\title{
REVIEW ARTICLE OPEN The mechanisms of sorafenib resistance in hepatocellular carcinoma: theoretical basis and therapeutic aspects
}

\author{
Weiwei Tang ${ }^{1}$, Ziyi Chen ${ }^{2}$, Wenling Zhang ${ }^{3}$, Ye Cheng ${ }^{1}$, Betty Zhang ${ }^{4}$, Fan Wu ${ }^{1}$, Qian Wang $\mathbb{D}^{1}$, Shouju Wang ${ }^{5}$, Dawei Rong ${ }^{2}$, \\ F. P. Reiter ${ }^{6,7}$, E. N. De Toni ${ }^{6,7}$ and Xuehao Wang ${ }^{2}$
}

Sorafenib is a multikinase inhibitor capable of facilitating apoptosis, mitigating angiogenesis and suppressing tumor cell proliferation. In late-stage hepatocellular carcinoma (HCC), sorafenib is currently an effective first-line therapy. Unfortunately, the development of drug resistance to sorafenib is becoming increasingly common. This study aims to identify factors contributing to resistance and ways to mitigate resistance. Recent studies have shown that epigenetics, transport processes, regulated cell death, and the tumor microenvironment are involved in the development of sorafenib resistance in HCC and subsequent HCC progression. This study summarizes discoveries achieved recently in terms of the principles of sorafenib resistance and outlines approaches suitable for improving therapeutic outcomes for HCC patients.

Signal Transduction and Targeted Therapy (2020)5:87

; https://doi.org/10.1038/s41392-020-0187-x

\section{INTRODUCTION}

Hepatocellular carcinoma (HCC) is the second leading cause of cancer-related mortality globally and usually presents in patients with chronic liver inflammation associated with viral infection, alcohol overuse, or metabolic syndrome. ${ }^{1,2}$ Significant progress has been made in HCC prevention, diagnosis and treatment in the past. However, more than $50 \%$ of all HCC patients have a diagnosis at an advanced stage (Barcelona Clinic Liver Cancer stage B or higher), and $70 \%$ of patients relapse within the first 5 years of initial treatment. ${ }^{2}$ Early HCC is often resectable, but advanced HCC often requires sorafenib for systemic treatment in addition to local treatment with ablation, transarterial chemoembolization, or external irradiation. ${ }^{3,4}$

In a groundbreaking study, sorafenib, a multiple-target tyrosine kinase inhibitor (TKI) exhibited antiangiogenesis and antiproliferation effects and extended total median survival in advanced HCC patients. ${ }^{5}$ Sorafenib suppresses tumor cell proliferation by inhibiting Raf-1, B-Raf, and kinase activity in the Ras/Raf/MEK/ ERK signaling pathways. In addition, sorafenib is capable of targeting platelet-derived growth factor receptor (PDGFR- $\beta$ ), vascular endothelial growth factor receptor (VEGFR) 2, hepatocyte factor receptor (c-KIT), and other proteins to inhibit tumor angiogenesis. ${ }^{6}$ In two significant clinical trials, Asia-Pacific and Sorafenib HCC assessment randomized protocol (SHARP), sorafenib was effective in improving the outcomes of HCC patients in the late stage, initiating a period of robust clinical research.,8 Since 2017, one large phase III trial has suggested noninferiority of lenvatinib compared with sorafenib in the first-line setting. Furthermore, regorafenib, cabozantinib, and ramucirumab have received approval as second-line treatments after sorafenib. ${ }^{9-12}$ Checkpoint inhibitors have also opened new strategies for the treatment of $\mathrm{HCC}^{13,14}$ Recently reported results from the IMbrave150 study (NCT03434379) show potential for the combination of atezolizumab with bevacizumab to expand the treatment options in first-line therapy for $\mathrm{HCC}^{15}$ However, immunotherapy for HCC has not yet been approved in China or Germany. Sorafenib remains a cornerstone treatment in HCC that is supported by robust evidence and clinical experience.

Only approximately $30 \%$ of patients can benefit from sorafenib, and this population usually acquires drug resistance within 6 months. ${ }^{16}$ Adverse events identified in patients administered sorafenib mainly included gastrointestinal, physical or skin diseases (e.g., hand and foot skin reactions, weight loss, and diarrhea). In serious cases, sorafenib can cause high blood pressure and abdominal pain, leading to treatment discontinuation. ${ }^{17}$ Accordingly, the sorafenib resistance mechanisms should be clarified. Recent studies suggest a role of epigenetics, transport processes, regulated cell death, and the tumor microenvironment in the initiation and development of sorafenib resistance in HCC. This study summarizes discoveries achieved recently in terms of the principles of sorafenib resistance and outlines approaches suitable for improving therapeutic outcomes for HCC patients.

\section{EPIGENETIC REGULATION AND SORAFENIB RESISTANCE IN HCC}

Epigenetic modifications can change the expression states of genes without changing DNA sequences, and some modifications

\footnotetext{
${ }^{1}$ Department of General Surgery, Nanjing First Hospital, Nanjing Medical University, Nanjing, China; ${ }^{2}$ Hepatobiliary/Liver Transplantation Center, The First Affiliated Hospital of

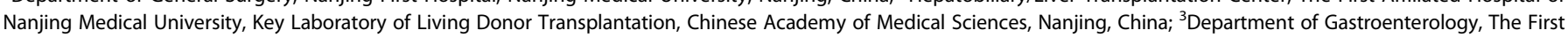

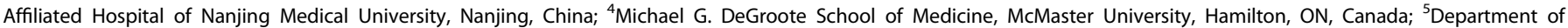

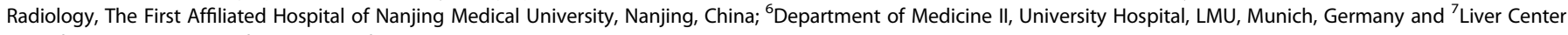
Munich, University Hospital, LMU, Munich, Germany

Correspondence: F P. Reiter (Florian.Reiter@med.uni-muenchen.de) or E Toni (Enrico.deToni@med.uni-muenchen.de) or Xuehao Wang (wangxh@njmu.edu.cn) These authors contributed equally: Weiwei Tang, Ziyi Chen, Wenling Zhang
}

Received: 13 January 2020 Revised: 14 March 2020 Accepted: 26 April 2020

Published online: 10 June 2020 
can be inherited. ${ }^{18}$ In some cases, epigenetic changes are dynamic and respond to environmental stimuli. Epigenetic mechanisms regulate different physiological processes that occur in living organisms, including cell proliferation and differentiation. ${ }^{19,20} \mathrm{~A}$ deeper understanding of epigenetic modifications associated with HCC could provide the basis for developing innovative approaches to treat this disease. In this context, we will describe the different types of epigenetic mechanisms and their involvement in the resistance of HCC to sorafenib (Table 1$).^{21-53}$

\section{Noncoding RNA-based mechanisms}

Increasing evidence indicates that noncoding RNAs (ncRNAs), including long noncoding RNAs (IncRNAs) and microRNAs (miRNAs), are critical for the development of sorafenib resistance in HCC (Fig. 1). Small nucleolar RNA host gene 3 (SNHG3) had significantly higher expression in highly metastatic HCC cells than in poorly metastatic HCC cells and induced epithelialmesenchymal transition (EMT) through miR-128/CD151 cascade activation to produce sorafenib resistance. ${ }^{28}$ SNHG16 was reported to be upregulated in HepG2 sorafenib-resistant (SR) cells, and SNHG16 knockdown increased the sensitivity of HepG2 SR cells to sorafenib in vitro and in vivo. Mechanistic studies indicated that SNHG16 could be an endogenous sponge for miR140-5p in HepG2 cells. Overexpression of miR-140-5p also made HepG2 SR cells more susceptible to sorafenib, and the influences exerted by SNHG16 knockdown on sorafenib resistance might be inhibited by miR-140-5p inhibitors. ${ }^{29}$ Overexpression of miR-591 has been reported to inhibit colony formation, as well as drug resistance, including sorafenib resistance, through the inhibition of the expression of far upstream elemental binding protein 2 (FBP2) via the phosphoinositide 3 kinase/Akt/mammalian target of the rapamycin axis. ${ }^{42}$ MiR-622 is significantly downregulated in $\mathrm{HCC}$ and functionally targets Kirsten rat sarcoma (KRAS), whose inhibition significantly inhibits RAF/ERK and PI3K/AKT signaling and resensitizes sorafenib-resistant cells. ${ }^{43}$ These studies indicate that ncRNAs may represent a medical treatment approach to overcome sorafenib resistance in HCC. However, further research is needed to determine whether ncRNAs are drivers or passengers in tumor progression, as numerous ncRNAs have been reported to be dysregulated in cancer progression. In addition, current studies have focused only on the role of IncRNAs as miRNA sponges in regulating target gene expression and mediating sorafenib resistance in HCC. Previous research indicates that IncRNAs have more mechanisms, such as binding to proteins regulating protein translation, interfering with the expression of genes encoding adjacent proteins, and forming complexes with proteins to regulate gene transcription, which are areas in need of further exploration. $^{54,55}$ Further studies of clinical trials are urgently needed to promote ncRNA-based therapeutic interventions beneficial to HCC patients, which may offer treatment avenues for sorafenib resistance.

\section{Methylation}

Methylation refers to the process of catalytically transferring methyl groups from active methyl compounds (such as Sadenosylmethionine) to other compounds. Examples consist of RNA methylation, histone methylation, and DNA methylation. ${ }^{56}$ Aberrant methylation causes gene expression to be changed, resulting in cancerous features. ${ }^{57}$ Wang et al. ${ }^{49}$ reported that the MORC2-NF2/KIBRA axis is critical to maintain sorafenib resistance and oncogenicity of HCC cells in vitro and in nude mice. MORC2 complexes with DNA methyltransferase 3A (DNMT3A) on the promoters of NF2 and KIBRA, causing DNA hypermethylation and transcriptional inhibition. Accordingly, under physiological and pathological conditions, NF2 and KIBRA are key targets of MORC2 for the regulation of fusion-induced Hippo signaling activation and contact inhibition of cell growth. Abeni et al. ${ }^{52}$ used medical chip technology to find 1230 differentially methylated genes in sorafenib-treated HA22T/VGH cells. After sorafenib treatment, oncogenes tend to be hypermethylated, while tumor suppressor genes tend to be hypomethylated. In addition, the IncRNA H19 is an example of maternal expression and epigenetic regulation of imprinted gene products and is believed to promote or inhibit tumors. In sorafenib-resistant cell lines, the promoter methylation of the $\mathrm{H} 19$ gene differs noticeably from that in sensitive cells. Overexpression of H19 sensitizes sorafenib-resistant cells by reducing cell proliferation after sorafenib treatment. A model of $\mathrm{H} 19$ knockout mice suggested that $\mathrm{H} 19$ promoted tumor progression and tumor cell proliferation after treatment with the carcinogen diethylnitrosamine (DEN), while administration of insulin-like growth factor 2 (IGF2) had no effect. Therefore, H19 may be a target for future strategies to overcome sorafenib resistance in HCC. ${ }^{23}$ Together, this evidence suggests that sorafenib may influence the methylation levels of cancer-related genes in HCC, which are valuable in tracing sorafenib resistance.

\section{TRANSPORT AND SORAFENIB RESISTANCE IN HCC}

Sorafenib resistance involves ATP binding box (ABC) transporters, which reduce the effectiveness of chemotherapy by pulling drugs out of cancer cells and negatively affect the outcomes of anticancer therapies. ${ }^{58,59}$ In addition, exosomes are carriers of intercellular information and regulators of the tumor microenvironment. In normal cells, exosomes remove adverse biomolecules, but this mechanism may be hijacked in cancer cells. For example, drug-resistant cancer cells can encapsulate therapeutic drugs in exosomes and transport them out of tumor cells. ${ }^{60,61}$ The mechanisms of the transport process and sorafenib resistance in HCC are reviewed below (Table 2). ${ }^{26,41,62-69}$

\section{$A B C$ transporters}

Several TKIs, including sorafenib, were found to interact with $A B C$ transporters. ${ }^{58}$ Such findings reveal a highly sophisticated situation in which TKls are likely to act as substrates or inhibitors, depending on specific pump expression and the type of drug coadministered, its affinity for transporters, and its concentration. The repositioning of TKIs as $A B C$ transporter antagonists opens up new avenues for anticancer therapy and clinical strategies aimed at counteracting drug resistance. Di Giacomo et al. ${ }^{62}$ investigated the ability of CRYO (the natural sesquiterpene component of many essential oils) to inhibit $A B C$ pumps and improve the response of HCC cells to sorafenib at nontoxic doses. They obtained a clonal subfamily from human HCC cells exhibiting increased multidrug resistance (MDR) related to upregulation of MRP1 and MRP2. In addition, CRYO restrained sorafenib degradation, facilitated its intracellular accumulation and strengthened its cytotoxic response. Another study reported that COP9 signaling corset 5 (CSN5) was associated with sorafenib resistance in HepG2/S HCC cells. After CSN5 silencing, resistance to sorafenib was reversed, and several resistance-related proteins (including $A B C B 1, A B C C 2$, and $A B C C_{2}$ ) were downregulated. ${ }^{63}$ Furthermore, $A B C C 1-3$ expression increased in SR cells. Enhanced SR cell migration and invasion and an increased ratio of CD44+ to CD44+ CD133+ cells were observed in SR cells. ${ }^{64}$

\section{Exosomes}

Exosomes, which are small extracellular vesicles (EVs), contribute to cell-to-cell communication and have emerged as a therapeutic target. $^{70-72}$ LincRNA-VLDLR (linc-VLDLR) showed significant upregulation in malignant liver cells. Exposure of HCC cells to various anticancer agents (e.g., sorafenib) increased the expression of lincVLDLR in cells and in EVs released from these cells. Incubation with EVs downregulated chemotherapy-triggered cell death and increased linc-VLDLR expression in recipient cells. In addition, knockdown of linc-VLDLR reduced ABCG2 (ATP binding cassette, 


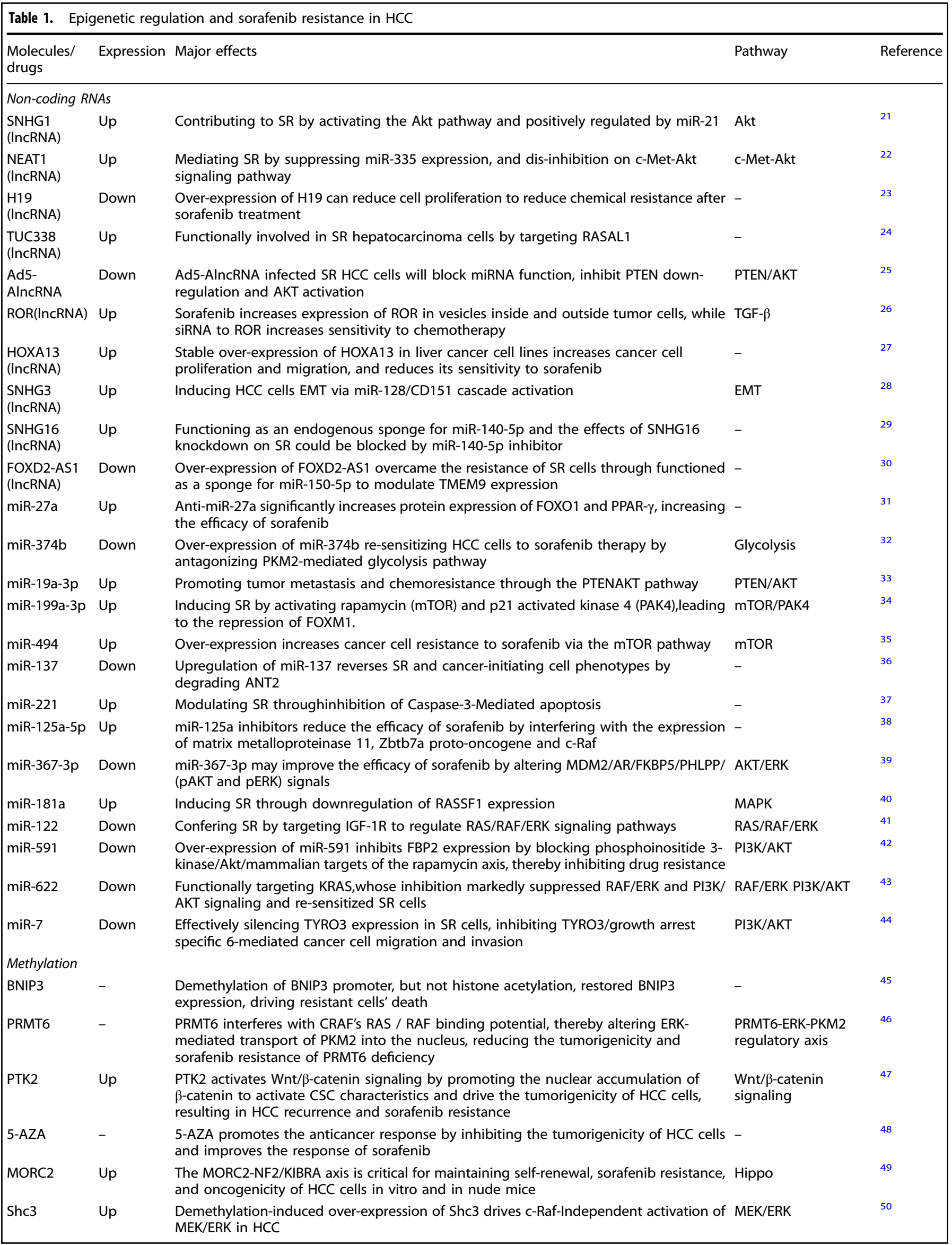


Table 1. continued

\begin{tabular}{|c|c|c|c|c|}
\hline $\begin{array}{l}\text { Molecules/ } \\
\text { drugs }\end{array}$ & Expression & Major effects & Pathway & Reference \\
\hline PD-L1 & Up & $\begin{array}{l}\text { Targeting the NFKB/PDL1/STAT3/DNMT1 axis can lead to dual inactivation of PD-L1 and } \\
\text { DNMT1 inhibitors, reducing cancer cell resistance to sorafenib }\end{array}$ & $\begin{array}{l}\text { NFKB/PDL1/STAT3/ } \\
\text { DNMT1 axis }\end{array}$ & 51 \\
\hline MDIG & Up & $\begin{array}{l}\text { MDIG affects the level of p21 (CIP1/WAF1) and the resistance of cancer cells to sorafenib } \\
\text { through the expression of H3K9me3 in HCC }\end{array}$ & H3K9me3/p21 & 53 \\
\hline
\end{tabular}

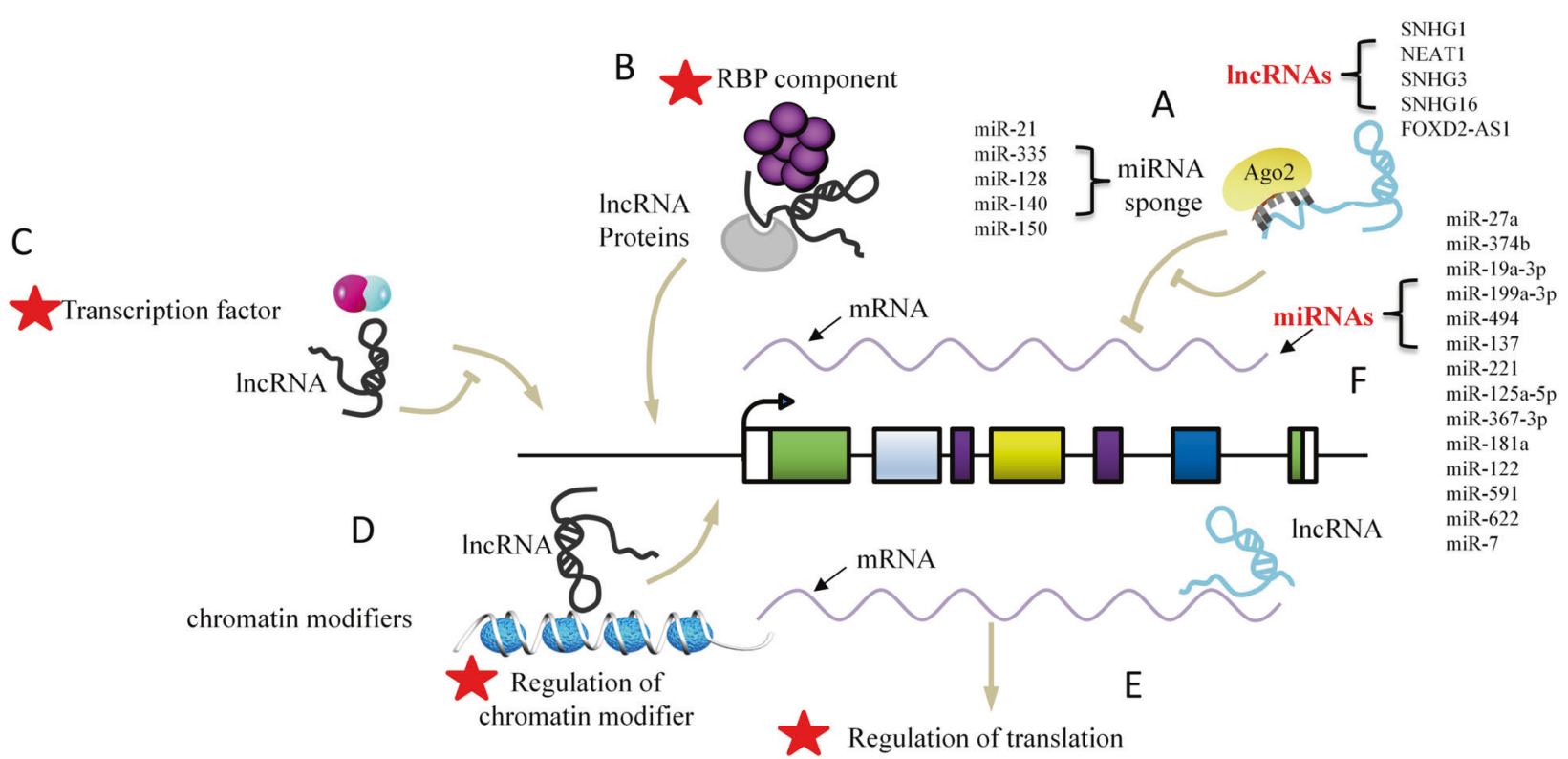

Fig. 1 Molecular mechanisms by which IncRNAs and miRNAs modulate sorafenib resistance. a LncRNAs can act as a "sponge" of miRNAs, competitively binding miRNAs, and thus affect the regulation of miRNAs on downstream target genes. The figure lists the IncRNAs and corresponding sponged miRNAs associated with SR. b As a scaffold or bridge for protein interaction, IncRNAs affect the formation of protein multimers and regulate protein activity. c As an RNA decoy, IncRNAs bind to transcription factors and interfere with their binding to the gene promoter region, thereby regulating transcription. $\mathbf{d}$ LncRNAs recruit chromatin modifiers to alter the level of chromatin modification, thereby affecting gene transcription and expression. e LncRNAs bind to mRNA and inhibit translation. $f$ MiRNAs have the ability to degrade mRNA and prevent mRNA translation. The figure lists the miRNAs associated with SR. Note: A pentagram indicates no relative report with SR in HCC

subfamily $\mathrm{G}$ member 2) expression, and overexpression of this protein reduced the effect of linc-VLDLR knockdown on sorafenibinduced cell death. ${ }^{67}$ Apart from IncRNAs, miRNAs can also be carried in exosomes, and miR-122-transfected adipose tissue mesenchymal stem cells (AMSCs) have been shown to efficiently package miR-122 in secreted exosomes, probably mediating AMSC and HCC cell miR-122-related communication processes, thus making HCC cells sensitive to sorafenib by altering the expression of miR-122 target genes. Intratumor injection of miR122-exo noticeably improved the antitumor performance of sorafenib in HCC in vivo. ${ }^{41} \mathrm{Li}$ et al. ${ }^{68}$ showed that the combination of si-GRP78-modified exosomes and sorafenib could target GRP78 in HCC cells and restrain cancer cells from growing and invading. Therefore, si-GRP78-altered exosomes can sensitize sorafenibresistant cancer cells to reverse drug resistance. Dendritic cells (DCs) are critical to both primary and secondary immune responses; thus, DC-derived exosomes are candidates for specific cancer treatment. Shi et al. ${ }^{69}$ found that regulatory T cells in tumor tissues and sorafenib treatment of HCC mice in situ increased programmed death ligand 1 (PD-L1) expression. When DCs were pulsed with exosomes from tumor cells, the number of regulatory
$\mathrm{T}$ cells decreased, and the number of $\mathrm{CD}^{+} \mathrm{T}$ cells increased. When anti-programmed death receptor-1 (PD-1) antibody (PD-1 $\mathrm{Ab}$ ) was added, the exhausted $\mathrm{CD}^{+} \mathrm{T}$ cells were restored, while the regulatory $T$ cell number remained unchanged. Because exosomes are involved in the development of many diseases, researchers have used exosomes as a therapeutic strategy, in which exosomes are loaded with therapeutic agents such as functional proteins, ncRNAs, and chemotherapy drugs (Fig. 2). The resistance mechanisms involving exosome-mediated crosstalk need to be addressed by future therapeutic strategies for advanced-stage HCC.

\section{REGULATED CELL DEATH (RCD) AND SORAFENIB RESISTANCE IN HCC}

RCD is used to describe the death of cells originating from the intracellular or extracellular microenvironment via molecular mechanisms when other adaptive responses cannot restore cell homeostasis, and RCD can be divided into different categories, including apoptosis, autophagic cell death, proptosis, ferroptosis, etc., according to the different mechanisms. ${ }^{73,74}$ It has been 


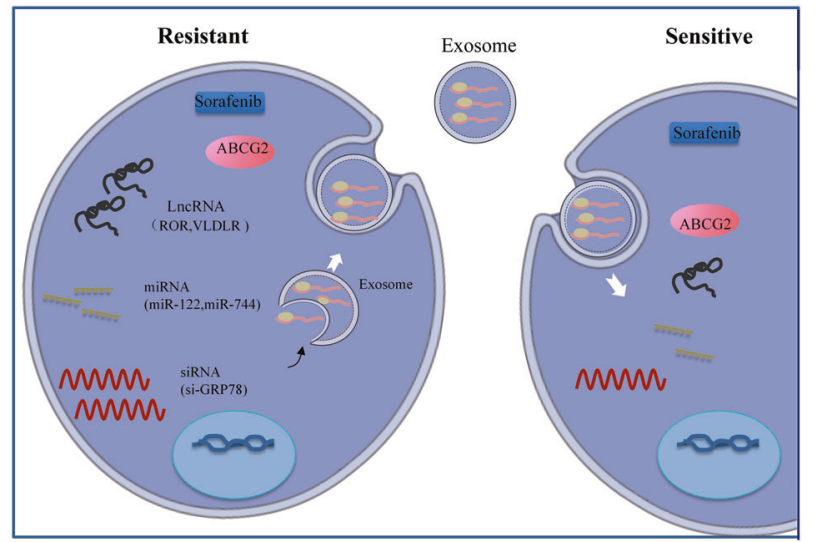

Fig. 2 Exosome application in HCC therapy. Exosomes derived from cancer cells can be used to deliver functional RNAs, including IncRNAs, siRNAs, miRNAs, and mRNAs

reported that RCD, especially autophagy and ferroptosis, is involved in sorafenib resistance in HCC (Table 3$){ }^{75-91}$

\section{Autophagy}

Autophagy is an important process leading to intracellular material turnover in eukaryotes. Some of the damaged proteins or organelles in this process are encapsulated in bilayered autophagic vesicles and sent to lysosomes for degradation and recycling. ${ }^{92,93}$ The effect exerted by autophagy in cancer cells plays a double-edged sword. ${ }^{94,95}$ Basic autophagy is a cancer suppressor that maintains genomic stability in normal cells, whereas activated autophagy promotes the survival of cancer cells under stress once cancer occurs. ${ }^{96}$ Autophagy is also thought to be an important mechanism for drug resistance by supporting the survival of tumor cells in the case of therapeutic and metabolic stress. ${ }^{97}$ Therefore, it is necessary to determine how to control cell growth, apoptosis and sorafenib resistance by changing autophagy levels, which could possibly improve the efficacy of sorafenib and the treatment of HCC. Activation of Akt is considered to account for the mediation of acquired resistance to sorafenib. Zhai et al. ${ }^{77}$ found that Akt inhibition reversed acquired resistance to sorafenib by transforming autophagy from a cell-protective effect to a system that promotes cell death. Moreover, sorafenib effectively reversed the activation of metformin-induced $\mathrm{mTORC} 2$ and enhanced the inhibitory effect of metformin on the mTORC1 and MAPK pathways in HCC cells. Pharmacological inhibition of autophagy sensitized HCC cells to apoptosis induced by metformin and sorafenib. ${ }^{79}$ Studies have also shown that patients exhibiting high ATG7 expression and an active autophagy state have poor prognosis after sorafenib treatment. ${ }^{80}$ Due to different autophagy responses to sorafenib, different HCC cell lines have different sensitivities to sorafenib. ${ }^{81}$ Animal models are seen as key tools in cancer research. A recent study has shown that ADRB2 signaling restrained autophagy through disruption of the Beclin1/VPS34/Atg14 complex in an AKT-dependent manner, causing HIF1a stabilization, reprogramming of glucose metabolism in HCC cells, and sorafenib resistance in DEN-induced HCC mouse models. ${ }^{82}$ Despite ample evidence that targeted autophagy processes represent potential therapeutic interventions for HCC (Fig. 3), there are many unresolved issues related to autophagy in sorafenib resistance. How do we selectively target autophagy mechanisms? How do we balance the dual effects of autophagy? What determines the threshold for autophagy to change from a survival mechanism to one that promotes apoptosis or autophagic death? The answers to these questions need to be further elucidated through research. 
Table 3. Regulated cell death and sorafenib resistance in HCC

\begin{tabular}{|c|c|c|c|c|}
\hline \multicolumn{5}{|l|}{ Autophagy } \\
\hline IRE1 & & $\begin{array}{l}\text { Sorafenib induces apoptosis and autophagy through endoplasmic reticulum stress, and further } \\
\text { induces autophagy independently of the MEK } 1 / 2 \text {-ERK } 1 / 2 \text { pathway. }\end{array}$ & MEK1/2-ERK1/2 & 75 \\
\hline SHP-1 & & $\begin{array}{l}\text { Silencing of SHP-1 by small interference RNA (siRNA) reduced the effect of sorafenib on P-STAT3 and } \\
\text { autophagy }\end{array}$ & $\begin{array}{l}\text { STAT3/Mcl-1/ } \\
\text { Beclin } 1\end{array}$ & 76 \\
\hline AKT & & $\begin{array}{l}\text { Inhibition of Akt reversed the acquired resistance to sorafenib by switching autophagy from a } \\
\text { cytoprotective role to a death-promoting mechanism }\end{array}$ & - & 77 \\
\hline $\mathrm{HDACls}$ & - & $\begin{array}{l}\text { Histone deacetylase inhibitors HDACls sensitized HCC cells to sorafenib treatment by regulating the } \\
\text { acetylation level of Beclin-1 }\end{array}$ & P53 & 78 \\
\hline Melatonin & - & $\begin{array}{l}\text { Melatonin increased the sensitivity of HCC to sorafenib by inhibiting autophagy through the PERK- } \\
\text { ATF4-Beclin1 pathway }\end{array}$ & PERK/ATF4/Beclin 1 & 79 \\
\hline \multirow[t]{2}{*}{ PSMD10 } & Up & $\begin{array}{l}\text { PSMD10/gankyrin induced autophagy to induce SR through cytoplasmic interaction with ATG7 and } \\
\text { nuclear transactivation of ATG7 expression }\end{array}$ & - & 80 \\
\hline & - & Sorafenib substantially increased phosphorylation of AMPK and consequently autophagy in Huh7 & AMPK & 81 \\
\hline ADRB2 & Up & $\begin{array}{l}\text { ADRB2 signals negatively regulate autophagy, stabilize HIF- } 1 \alpha \text {, reprogram glucose metabolism in HCC } \\
\text { cells, and acquire resistance to sorafenib }\end{array}$ & ADRB2 & 82 \\
\hline Capsaicin & - & $\begin{array}{l}\text { Capsaicin and sorafenib combination treatment inhibited the growth, invasion and metastasis of HCC } \\
\text { cells and induced autophagy in a synergistic manner }\end{array}$ & $\begin{array}{l}\text { EGFR PI3K/Akt/ } \\
\text { mTOR }\end{array}$ & 84 \\
\hline CD24 & Up & $\begin{array}{l}\text { CD24-related sorafenib resistance was accompanied by the activation of autophagy and can be } \\
\text { blocked by the inhibition of autophagy }\end{array}$ & mTOR/AKT & 85 \\
\hline $\operatorname{miR}-21$ & Up & $\begin{array}{l}\text { Inhibiting miR- } 21 \text { enhances the efficacy of sorafenib in the treatment of sorafenib-resistant HCC } \\
\text { tumors, and reduces sorafenib resistance }\end{array}$ & AKT/PTEN & 86 \\
\hline $\operatorname{miR}-423-5 p$ & Up & $\begin{array}{l}\text { Promoting autophagy in cancer cells and was increased in serum from HCC patients treated with } \\
\text { sorafenib }\end{array}$ & - & 87 \\
\hline \multicolumn{5}{|c|}{ : } \\
\hline AIFM2 & - & AIFM2 blocks ferroptosis independent of ubiquinol metabolism. & - & 88 \\
\hline ZFP36/TTP & - & $\begin{array}{l}\text { RNA-binding protein ZFP36/TTP protects against ferroptosis by regulating autophagy signaling } \\
\text { pathway in hepatic stellate cells }\end{array}$ & - & 89 \\
\hline
\end{tabular}

\section{Ferroptosis}

Ferroptosis refers to a nonapoptotic, RCD procedure involving the abnormal metabolism of lipid oxides in cells catalyzed by iron ions or iron enzymes and has been identified recently. In this process, various inducers break the cell redox balance and produce considerable lipid peroxidation products, thereby triggering cell death. A growing body of research shows that the relationships between ferroptosis and cancer are significantly complex and that ferroptosis holds promise as a novel cancer treatment. ${ }^{98}$ The fact that sorafenib induces ferroptosis, which promotes sorafenib resistance adds to the complexity of sorafenib's antitumor mechanism in HCC (Fig. 4). ${ }^{98}$ A recent study reported that the depletion of intracellular iron stores realized through the iron chelator deferoxamine (DFX) strikingly protected HCC cells from the cytotoxic effects of sorafenib. Moreover, they identified that DFX did not prevent sorafenib from reaching its intracellular target kinases. Instead, the depletion of intracellular iron stores prevented sorafenib from inducing oxidative stress in HCC cells.99 ${ }^{99}$ Beyond that, Lachaier et al. ${ }^{100}$ reported that sorafenib induced ferroptosis in different cancer cell lines. Compared to other kinase inhibitors, sorafenib was the only drug that displayed ferroptotic efficacy. From a mechanism of action perspective, Sun et al. ${ }^{90}$ found that metallothionein (MT)$1 \mathrm{G}$ was a critical regulator and promising therapeutic target of sorafenib resistance in human HCC. The expression of MT-1G messenger RNA and protein was noticeably triggered by sorafenib but not by other clinically relevant kinase inhibitors. The activation of the transcription factor nuclear factor erythroid 2-related factor 2 , but not of p53 and hypoxia-inducible factor 1-alpha, was critical to induce MT-1G expression following sorafenib treatment. The molecular mechanisms of MT-1G in sorafenib resistance participate in the suppression of ferroptosis, a novel form of altered cell death. Knockdown of MT-1G by RNA interference increased glutathione depletion and lipid peroxidation, which contributed to sorafenibinduced ferroptosis. According to another study, ELAVL1 upregulation, ferritinophagy activation, and ferroptosis induction occurred in primary human hepatic stellate cells (HSCs) from the collected human liver tissues. ${ }^{91}$ In conclusion, sorafenib-induced ferroptosis may be an effective mechanism for inducing HCC cell death, regardless of its inhibitory effect on kinases.

TUMOR ENVIRONMENT AND SORAFENIB RESISTANCE IN HCC

Cancer metastasis, invasion and growth are affected by the tumor microenvironment, which is comprised of various nonmalignant stromal cells. A sophisticated and multidirectional interplay between immune or nonimmune stromal cells and tumor cells during HCC development and progression has been recently shown (Table 4). ${ }^{101-122}$ 

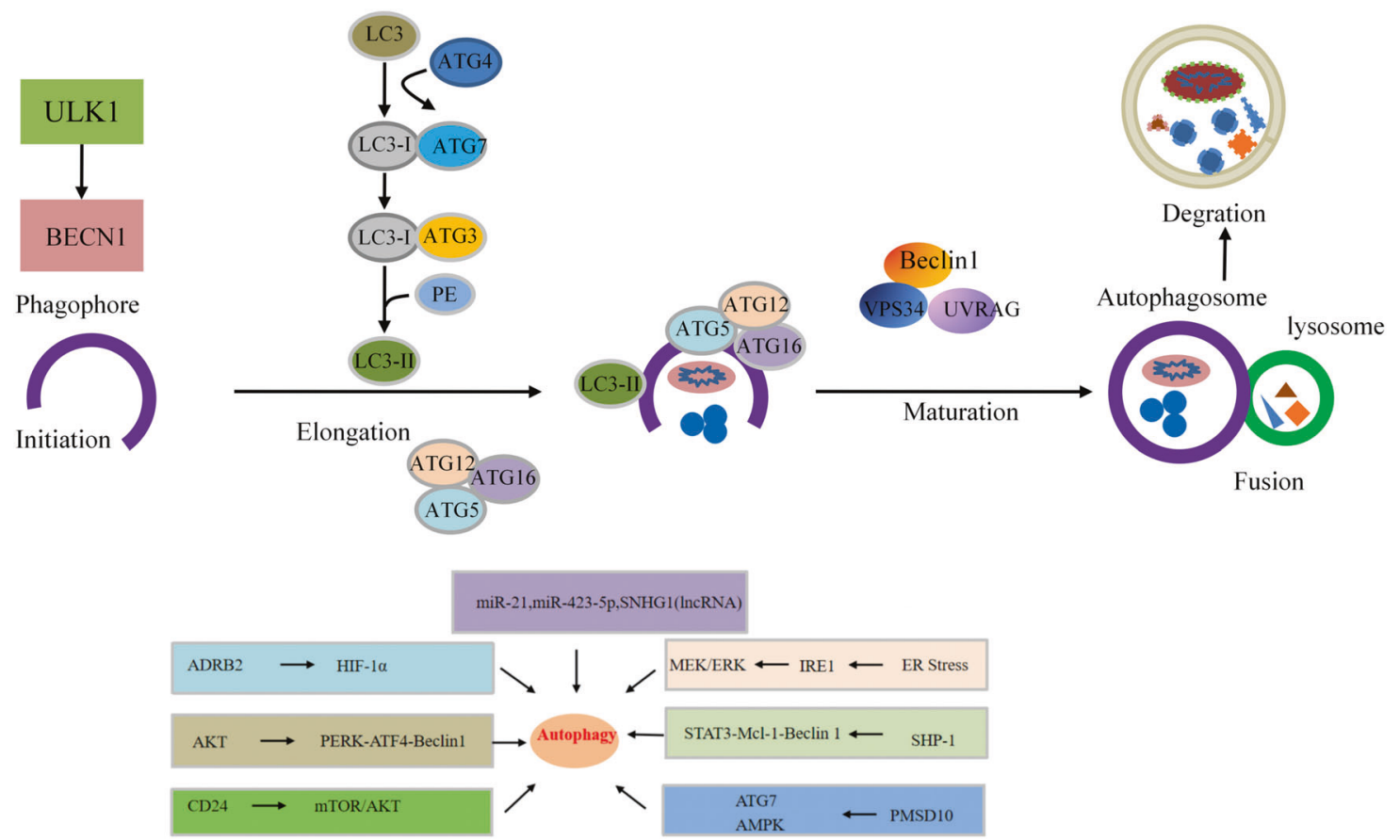

Fig. 3 Schematic diagram of autophagy flux. Autophagy is initiated (primed) by the nucleation of a membrane or phage. This process is initiated by the ULK1-Atg13-FIP200 complex. The membrane is then elongated to engulf the cytoplasmic component (elongation). The elongation of the phagocytic membrane depends on the Atg5-Atg12-Atg16 and LC3 coupling systems. At a later stage of autophagosome formation, LC3-II is localized to the elongated barrier membrane, while the Atg5-Atg12-Atg16 complex dissociates therefrom. Finally, the barrier membrane is closed to form autophagosomes. After autophagosome formation, lysosomes fuse with autophagosomes to form autolysosomes (autophagosome-lysosome fusions). The lysosomal hydrolase degrades the content (degradation) in the autophagosome. Beclin 1-VPS34-UVRAG complex positively regulates fusion and degradation

Hypoxia

Hypoxia in HCC drives angiogenesis through a vascular endothelial growth factor (VEGF)-producing process and hypoxia-inducible factor 1 (HIF-1a) activation. Therefore, the antiangiogenic effect of sorafenib results from blockade of the HIF-1a/VEGF pathway. ${ }^{123,124}$ Sorafenib suppresses the synthesis of hypoxia-inducible factor 1 (HIF-1a), resulting in a decrease in VEGF expression and tumor angiogenesis in $\mathrm{HCC}^{125}$ In addition, acquired sorafenib resistance and an anoxic microenvironment show an interesting correlation. Continuous sorafenib treatment results in inhibition of the tumor's antiangiogenic activity and subsequent hypoxia within the tumor, which facilitates the selection of resistant cell clones to adapt to oxygen and nutrition deficits, thereby limiting the efficiency of sorafenib. A study by Liang et al. ${ }^{101}$ reported that hypoxia induced by continued sorafenib treatment conferred sorafenib resistance in HCC via HIF-1a and NF-KB activation. EF24, a molecule having structural similarity to curcumin, overturned sorafenib resistance via VHL-dependent HIF-1a degradation and NF-KB inactivation. Prieto-Domínguez et al. ${ }^{106}$ found that the coadministration of melatonin and sorafenib decreased the expression of HIF-1a mitophagy targets and restrained the formation of autophagosomes and subsequent colocalization of mitochondria and lysosomes. Melatonin enhanced sensitivity to sorafenib in Hep3B cells and blocked the synthesis of HIF-1a, thus preventing the protective cell phagocytosis caused by the hypoxic microenvironment, which is an important part of the multifactor mechanism responsible for the failure of chemotherapy. In addition, miR-338$3 p$ was shown to inhibit HCC tumor growth and sensitize HCC cells to sorafenib by downregulating HIF-1a. ${ }^{103}$ Galectin-1 has been reported as a predictive marker of sorafenib resistance and a downstream target of the AKT/mTOR/HIF-1a signaling pathway. ${ }^{126}$ Considering the feedback system between HIF-1a and HIF-2a subunits, we believe that sorafenib treatment is likely to upregulate HIF-2a by inhibiting HIF-1a, enhancing sorafenib resistance and tumor growth. ${ }^{105,127}$ It has also been confirmed that the increase in HIF-2a triggered by sorafenib contributes to resistance through the activation of the TGF-a/EGFR pathway. ${ }^{107}$ Another study found that the HIF-2a inhibitor PT-2385 significantly enhanced sorafenib efficacy by suppressing HIF-2a, increasing androgen receptor (AR) and suppressing downstream pSTAT3/pAKT/pERK pathways. ${ }^{108}$ These studies endorse the existing relationship between high HIF expression and resistance to sorafenib (Fig. 5), demonstrating that hypoxia evidently impacts sorafenib therapy and suggesting that inducing hypoxia is a promising approach to overcoming resistance.

Immune microenvironment

Tumor immune escape has garnered interest in the development of tumor immunotherapy strategies, which have proved to be effective for some malignant neoplasms. ${ }^{128}$ Tumor-associated macrophages (TAMs) restrain antitumor immunity and facilitate tumor progression by expressing cytokines and chemokines. ${ }^{128}$ Current clinical trials of therapeutic drugs that promote phagocytosis or inhibit survival, proliferation, transport or polarization of TAMs have shown improved tumor prognosis. In HCC, Yao et al. ${ }^{113}$ reported that a natural product from Abies georgei, which is termed 747 and is related in structure to kaempferol, exhibited sensitivity and selectivity as a CCR2 antagonist. 747 enhanced the therapeutic efficacy of low-dose sorafenib without obvious toxicity by elevating the numbers of intratumoral $\mathrm{CD}^{+}{ }^{+} \mathrm{T}$ cells and increasing the death of tumor cells. Chen et al. ${ }^{114}$ found that sorafenib increased the numbers of $\mathrm{F} 4 / 80^{+}$TAMs and $\mathrm{CD} 11 \mathrm{~b}^{+} \mathrm{Gr}$ $1^{+}$and $\mathrm{CD}_{4} 5^{+} \mathrm{CXCR}^{+}$myeloid cells in both HCA-1 and $\mathrm{JHH}-7 \mathrm{HCC}$ models. Moreover, sorafenib treatment resulted in an increase in the fraction of tumor-infiltrating $\mathrm{CD}^{+} \mathrm{CD}_{2} 5^{+} \mathrm{FoxP}^{+}$regulatory $T$ cells (Tregs) in HCA-1 tumors. Using AMD3100 to inhibit the 


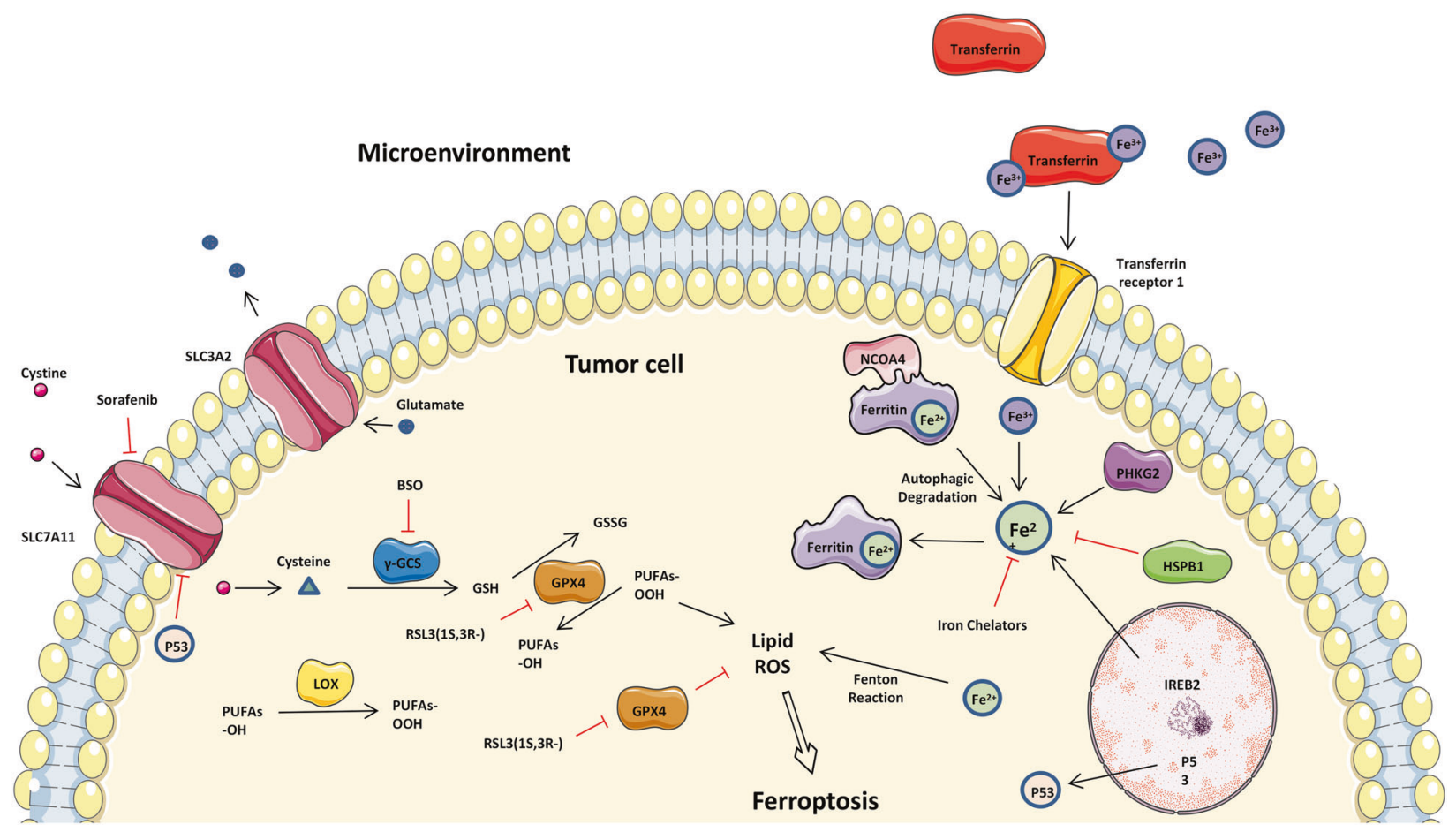

Fig. 4 The mechanisms of ferroptosis and sorafenib resistance. The key factor leading to ferroptosis are ROS, which are produced by iron accumulation and lipid peroxidation. This figure shows the relevant pathways that regulate iron and lipid peroxidation. SLC3A2 solute carrier family 3 member 2, SLC7A11 solute carrier family 7 member 11, BSO L-buthionine-sulfoximine, GPX4 glutathione peroxidase 4, PUFA polyunsaturated fatty acids, LOX lipoxygenase, NCOA4 nuclear receptor coactivator 4, PHKG2 phosphorylase kinase G2, HSPB1 heat shock protein family B (small) member 1, IREB2 iron-responsive element-binding protein 2

stromal cell-derived 1 receptor (C-X-C receptor type 4 or CXCR4) can prevent polarization of the immunosuppressive microenvironment after sorafenib treatment, inhibit tumor growth, reduce lung metastasis, and improve survival. In addition, studies have demonstrated that $M 2$, but not $M 1$, macrophages maintain tumor growth and metastasis by secreting hepatocyte growth factor (HGF), thereby significantly increasing tumor resistance to sorafenib. HGF activates the HGF/c-Met, ERK1/2/MAPK and PI3K/ AKT pathways in tumor cells. In vivo M2 tumor-associated macrophages accumulate more in sorafenib-resistant tumors than in sorafenib-sensitive tumors and produce large amounts of HGF. HGF can attract more macrophages from the surrounding area and regulate $\mathrm{M} 2$ macrophage distribution and feedback to enhance the sorafenib resistance of liver cancer. ${ }^{115}$ Neutrophils are capable of promoting or inhibiting tumor progression via the release of cytokines, which is determined by the tumor microenvironment. Factors generated by tumor-associated neutrophils (TANs) and their effects on tumor progression have not been elucidated. Zhou and colleagues ${ }^{116}$ explored the roles of TANs in the progression of HCC using cell lines and immune cells isolated from patients. They found that in tumor-bearing mice, sorafenib increased the number of TANs as well as CCL2 and CCL17 levels in the tumor. HCC tissues treated with sorafenib before surgery contained more TANs than tissues not treated with sorafenib. In mice, TAN depletion and sorafenib administration suppressed tumor growth and neovascularization more significantly than sorafenib administration alone. Phosphorylated extracellular signaling-regulated kinase ( $p E R K$ ) has been proposed as a marker for predicting the response to sorafenib in HCC, but clinical support is mixed or even contradictory. Chen et al. ${ }^{117}$ found that the pERK expression level varied in different patients with liver nodules. Mouse and human HCC samples with low pERK expression showed noticeable increases in intratumor $\mathrm{CD}^{+}$ cytotoxic $\mathrm{T}$ lymphocytes with robust inflammatory infiltrating cells and expression of PD-1, suggesting that anti-PD-1 immunotherapy may supplement sorafenib in HCC patients by targeting sorafenib-resistant cancer cells and overcoming drug resistance. Zhu et al. ${ }^{129}$ reported a patient with advanced HCC who had a number of lung metastases that progressed during sorafenib treatment. SHR-1210 (an anti-PD-1 therapy) alone was used as a second-line treatment. Although lung metastasis did not decrease after 3 months of treatment, it declined noticeably and partially disappeared after 6 months of treatment. In addition, all lung metastases decreased continuously even after 17 months of treatment. Alpha-fetoprotein levels revealed a similar effect. After 19 months of follow-up, the patient was in good health. This suggests that SHR-1210 alone as a second-line therapy for HCC patients has a good antitumor effect. Modifying the tumor microenvironment with immune checkpoint blockade is an emerging and promising strategy in the field of HCC treatment (Fig. 6). The concept of immune checkpoint blockade is now being investigated to treat advanced tumors in adjuvant and neoadjuvant settings.

Viral reactivation

Chronic hepatitis B virus (HBV) and hepatitis C virus (HCV) infection are major causes of HCC in the Western and Eastern regions. ${ }^{130}$ The reactivation process of virus infection in the setting of chemotherapy and immunosuppression is likely to cause fulminant liver failure and death. ${ }^{131,132}$ Zuo et al. ${ }^{133}$ reported that a high viral load of HBV DNA is the critical correctable risk element in HCC recurrence. Researchers began to look at the correlations between sorafenib efficacy and viral reactivation, but opinions remain controversial. Two recently conducted studies reported that sorafenib blocked HCV infection by altering the viral entry step and the production of viral particles. In addition, sorafenib led to modification of claudin-1 expression and localization, which could partly be responsible for the anti-HCV effect. ${ }^{134,135}$ The opposite was shown in another study: combined application of sorafenib and interferon-a as an 


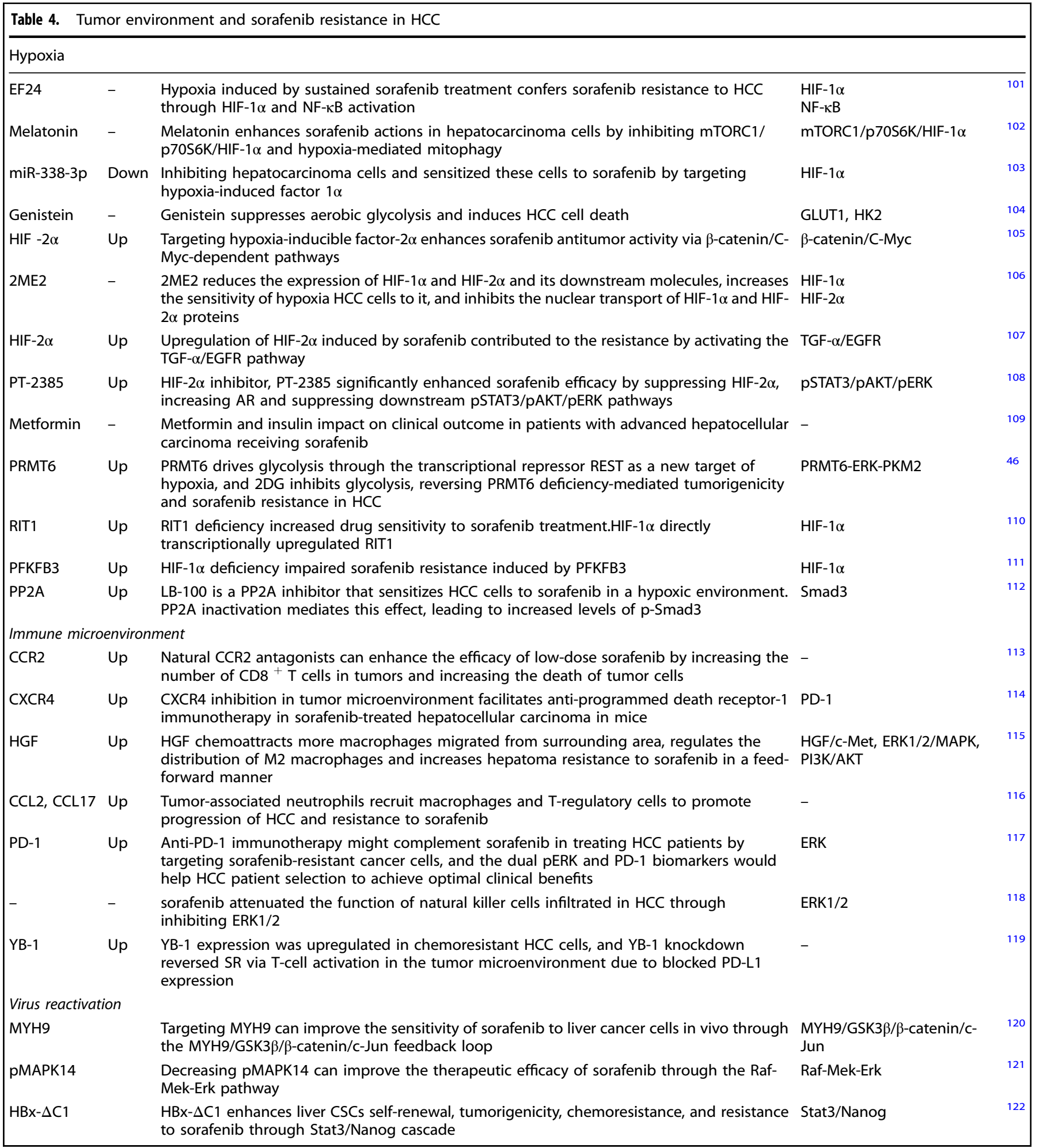

antiviral treatment was not beneficial. ${ }^{136}$ Moreover, Zhang and colleagues $^{137}$ reported that sorafenib promoted virus reactivation by significantly reducing the number of natural killer (NK) cells and inhibiting the reactivity of NK cells against HCC cells. Lin et al. ${ }^{120}$ reported that targeting MYH9 noticeably promoted the survival of HCC-bearing mice and promoted sorafenib sensitivity of HCC cells in vivo. HBV X protein (HBX) interacted with MYH9 and triggered its expression through the modulation of GSK3 $\beta$ /
$\beta$-catenin/c-Jun signaling. Witt-Kehati et al. $^{121}$ reported that inhibition of pMAPK14 overturned resistance to sorafenib in hepatoma cells with HBV. The phosphorylated form of the prooncogenic protein mitogen-activated protein kinase 14 (pMAPK14) was triggered in sorafenib-treated hepatoma cells and related to HBV X protein expression. Therefore, there is no consensus on the correlation between viral reactivation and sorafenib therapy. 


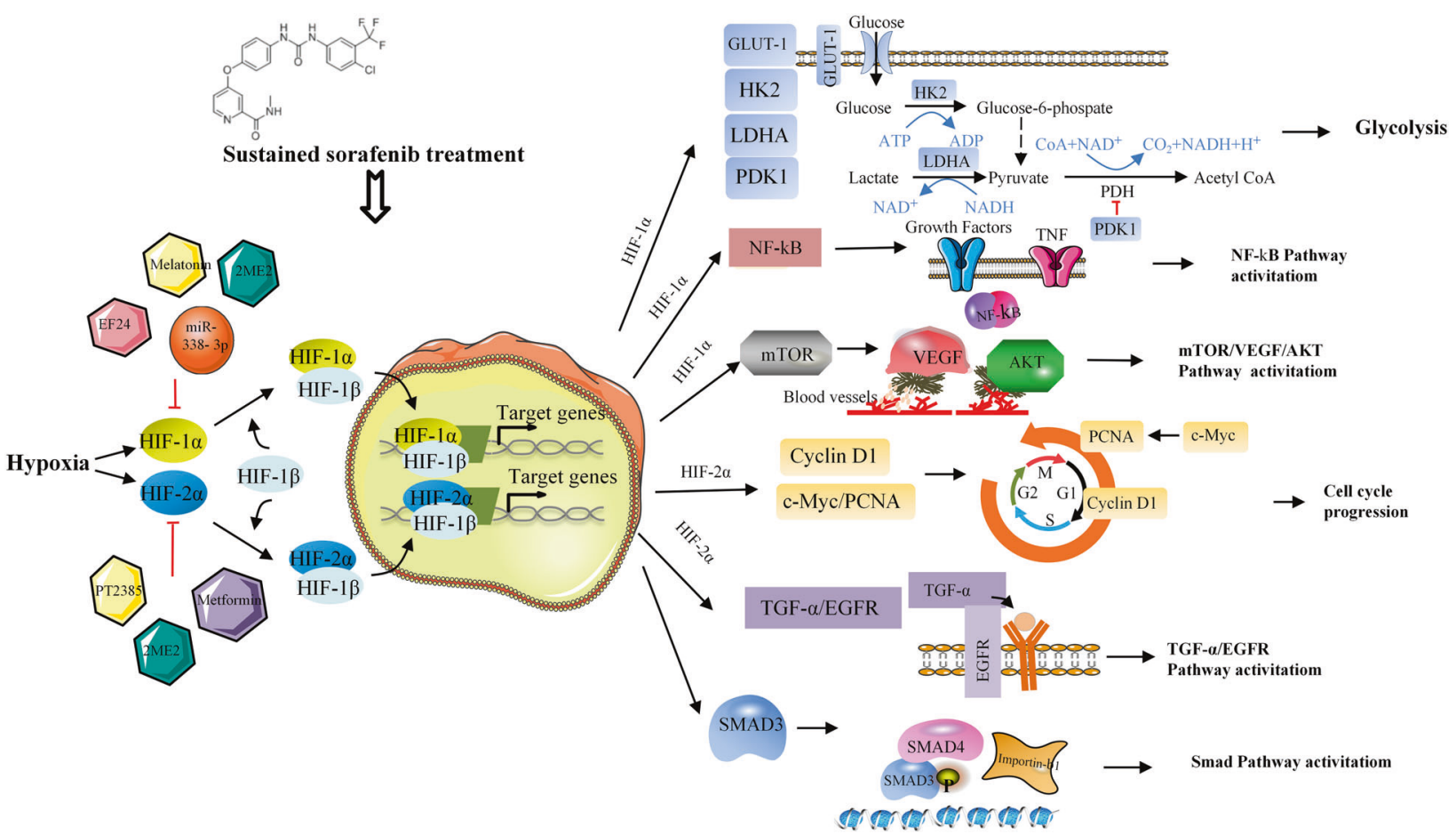

Fig. 5 Hypoxia-related sorafenib resistance mechanisms and strategies for targeting HIFs. Sustained sorafenib treatment enhances hypoxiainducible factors 1 alpha and 2 alpha, thereby promoting transcription of multiple genes involved in proliferation, glucose metabolism, angiogenesis, and different pathways, leading to sorafenib resistance. This resistance can be overcome by different small molecules or drugs inhibiting HIFs

\section{OTHER POSSIBLE MECHANISMS OF SORAFENIB RESISTANCE IN HCC}

Some diverse studies have indicated that sorafenib suppresses the EMT process. KPNA3, KIAA1199, and CDK1 were markedly elevated in SR cells and positively related to a high risk of recurrence and metastasis and advanced TNM stage in patients with HCC. All of them were found to trigger EMT and are involved in EGFR phosphorylation and the AKT-ERK signaling cascade. ${ }^{138-140}$ Blocking these genes may improve sorafenib antitumor responses, providing a rational combination treatment to increase sorafenib efficacy (Table 5). ${ }^{138,141-143}$ It has been recently shown that cancer stem cells (CSCs) also participate in therapeutic resistance in HCC. CSC markers act as predictors in the response to sorafenib. Overexpression of the CSC markers CD90 and CD133 in HCC was related to a poorer response to sorafenib than normal expression of these markers (Table 5). ${ }^{144}$ Protein tyrosine kinase 2 (PTK2) was found to activate CSC traits and drive tumorigenicity by facilitating the nuclear accumulation of $\beta$-catenin in HCC cells, leading to sorafenib resistance. ${ }^{144}$ Xiao et al. $^{145}$ reported that hypoxia is likely to enrich the HCC CSC population by changing AR/miR-520f-3p/SOX9 signaling, and targeting of this affected signaling by the small molecule miR$520 f-3 p$ could facilitate novel therapy to more effectively restrain HCC progression. These studies will help motivate researchers to explore sorafenib resistance and help develop effective strategies for the treatment of HCC in the clinic. Apart from this, several studies on sorafenib resistance in HCC have been reported (Table 5 ) ${ }^{146-148}$ however, the mechanism is not within the scope of this review. For example, Haga et al. ${ }^{146}$ found that overexpression of c-Jun contributed to sorafenib resistance in human hepatoma cell lines. The activation of JNK and high CD133 expression levels predicted an ineffective response to sorafenib in HCC. ${ }^{147}$ Downregulation of TGF- $\beta$ expression reversed the sorafenib resistance of HCC cells. ${ }^{148}$

\section{STRATEGIES TO OVERCOME SORAFENIB RESISTANCE IN HCC} Combination of cytotoxic chemotherapeutic agents

Novel combination strategies including sorafenib and cytotoxic drugs have been studied to overcome resistance to sorafenib and to more effectively treat intermediate and advanced HCC. Wang et al. $^{149}$ reported that sequential treatment with sorafenib and irinotecan was significantly better than monotherapy at inhibiting the growth of HepG2 xenografts. Sequential treatment with sorafenib and irinotecan noticeably elevated the levels of cleaved caspase-8, cleaved caspase-3, and PARP in HepG2 cells. Sorafenib inhibits p53 expression at both the mRNA and protein levels, which may facilitate cell cycle arrest and sensitization of tumor cells to irinotecan. Sequential therapy with sorafenib and irinotecan enhanced the efficacy of the two drugs alone in inducing apoptosis of HepG2 cells in vitro and inhibiting the growth of xenograft HepG2 cells in vivo. Although the addition of sorafenib to adriamycin did not significantly delay progression, the median survival time associated with sorafenib plus adriamycin was significantly longer than that associated with adriamycin alone in terms of overall survival and progression-free survival. ${ }^{150}$ In recent years, it has been reported that the liquid-gas phase transformation of sorafenib/doxorubicin-nanodroplets (SF/DOXNDs) can be used as cavitation nuclei to promote drug release and increase cell uptake after therapeutic ultrasound (TUS) irradiation. In addition, this strategy can also induce the apoptosis of HCC cells and suppress HCC cell proliferation, migration and invasion, suggesting that SF/DOX-ND combination treatment may be a lowside effect and effective treatment for HCC. ${ }^{151}$ In addition, it was found that GEMOX based on sorafenib as a first-line medical treatment followed by sorafenib as maintenance therapy exhibited high performance with manageable toxicity for HCC patients in the late stage. ${ }^{152}$ Regarding the mechanism, a recent study showed that NOD2 made HCC cells noticeably more susceptible to sorafenib, lenvatinib and 5-FU treatment by activating the AMPK 


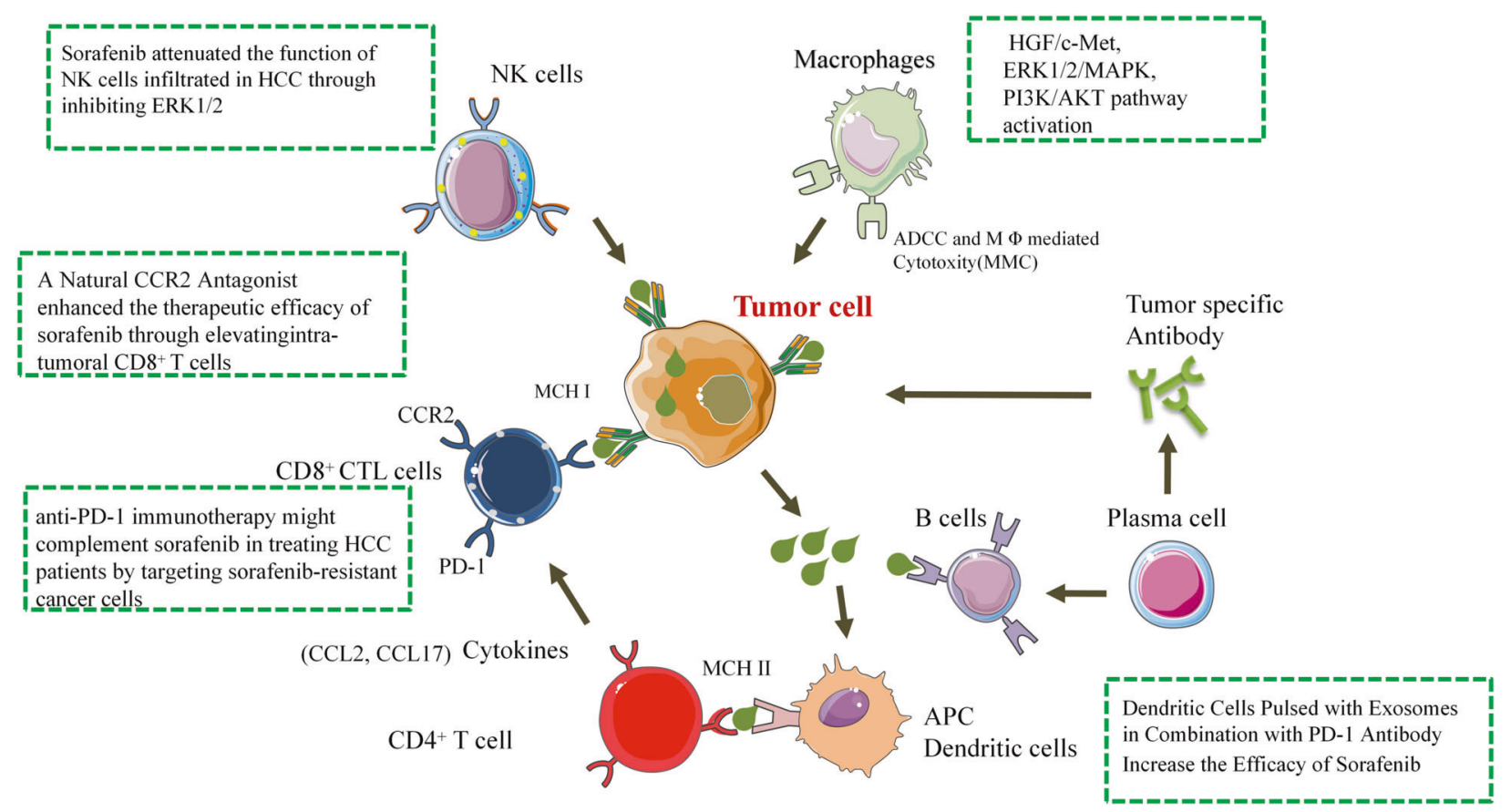

Fig. 6 Schematic diagram of the sorafenib resistance-mediated immune mechanism. CD8 ${ }^{+}$CTL cells, NK cells, DC cells, and macrophages have been confirmed to be involved in sorafenib resistance through different mechanisms

pathway to trigger apoptosis. ${ }^{153}$ Slit3 repression triggered chemoresistance to sorafenib, oxaliplatin and 5-FU via cyclin D3 and by enxtending survival and inhibiting $\beta$-catenin degradation. ${ }^{154}$ However, the different pharmacokinetic characteristics, hydrophobicity, and systemic toxicity of these drugs present serious challenges to the clinical application of this combination therapy. The efficacy and safety of some cytotoxic chemotherapy drugs are still being determined in phase III clinical trials, and the clinical use of cytotoxic chemotherapy drugs in combination with sorafenib requires further research.

Combination of molecular targeted agents

Targeting EGFR. EGF and EGFR are critical to the development of HCC, and strategies targeting EGFR are able to overcome sorafenib resistance. In clinical practice, in addition to anti-EGFR antibodies (e.g., cetuximab), there are also TKls targeting EGFR. According to some preclinical studies, cetuximab alone or in combination can inhibit HCC cell proliferation. ${ }^{155-157}$ Nevertheless, in one phase II study, cetuximab therapy did not show encouraging results. ${ }^{158}$ One study explored the performance of a combination of the anti-EGFR antibody cetuximab with oxaliplatin and capecitabine in late-stage HCC and showed that the capecitabine/oxaliplatin/cetuximab combination was tolerable, although there was a high rate of diarrhea. The combination was associated with only a modest response rate but a substantial a-fetoprotein (AFP) response and a high radiographically determined stable disease rate. The time to progression and overall survival were shorter than would be expected for treatment with sorafenib. ${ }^{159}$ In patients who received gemcitabine plus oxaliplatin (GEMOX) combined with cetuximab therapy, the response rate reached $20 \%$, while $40 \%$ of patients developed stable disease. ${ }^{160}$ Erlotinib is an EGFR-specific receptor tyrosine kinase inhibitor. Disappointingly, a recent phase III randomized, double-blind, placebo-controlled trial showed that sorafenib in combination with erlotinib had no beneficial effect on survival in late-stage HCC patients compared to sorafenib alone. ${ }^{161}$

Targeting PI3K/AKT/mTOR signaling. Sorafenib suppresses the Raf/ MAPK signaling pathway, whereas it can activate the PI3K/AKT pathway, suggesting an interaction between the MAPK/ERK pathway and the PI3K/AKT pathway. The potential compensation mechanism presented by the PI3K/AKT pathway can cause sorafenib resistance in HCC patients. ${ }^{162,163}$ Accordingly, by inhibiting the multiple pathways involved in HCC, a more effective survival outcome is likely to be achieved with a combination of targeted therapies. Liangtao Ye et al. ${ }^{164}$ found that copanlisib led to cell cycle arrest by affecting the cyclin D1/CDK4/6 signaling pathway, which significantly reduced cell activity and inhibited the colony forming process in various natural and sorafenib-resistant HCC cells. An increase in phosphorylated AKT was identified in cells being treated with sorafenib and was uniformly observed in 6 diverse clones of sorafenib-resistant cells that were not stimulated. Sorafenib plus copanlisib to treat HCC in the late stage is a reasonable potential therapy. Interestingly, the novel CDK4/6 inhibitors palbociclib and ribociclib, which were recently approved to treat hormone receptor-positive and HER2-negative breast cancer, both demonstrated antitumor activity in SR HCC cells and acted synergistically with sorafenib in HCC cell lines. Both agents induced cell cycle arrest in HCC cells that express Rb protein. ${ }^{165,166}$ Marozin et al. ${ }^{167}$ demonstrated that NSC74859, a specific inhibitor of signal transducer and activator of transcription 3 (STAT3), effectively inhibits the proliferation of HCC cells and is likely to be incorporated into vesicular stomatitis virus (VSV) oncolytic virus therapy. The protection of primary hepatocytes and nervous system cells from virus-triggered cytotoxic effects by NSC74859 increased the maximal tolerated dose of mouse VSV and enhanced the potential benefits of this combination therapy. The combination of sorafenib, which targets the PI3K/AKT/mTOR pathway, with PKI-587, which primarily targets the Ras/Raf/MAPK signaling pathway, outperformed single-agent therapy by blocking both signaling pathways. ${ }^{168}$ However, no evidence was found that sorafenib plus everolimus improves the efficacy compared with sorafenib alone. A combination of $5 \mathrm{mg}$ everolimus with full-dose sorafenib is feasible but has been shown to be more toxic than sorafenib alone. ${ }^{169}$

Combination with immunotherapeutic drugs

Immune checkpoint blockade is a promising treatment for HCC. Immunotherapy drugs cannot undergo liver metabolization, and HCC has moderate immunogenicity. For these reasons, from a 
Table 5. Other factores and sorafenib resistance in HCC

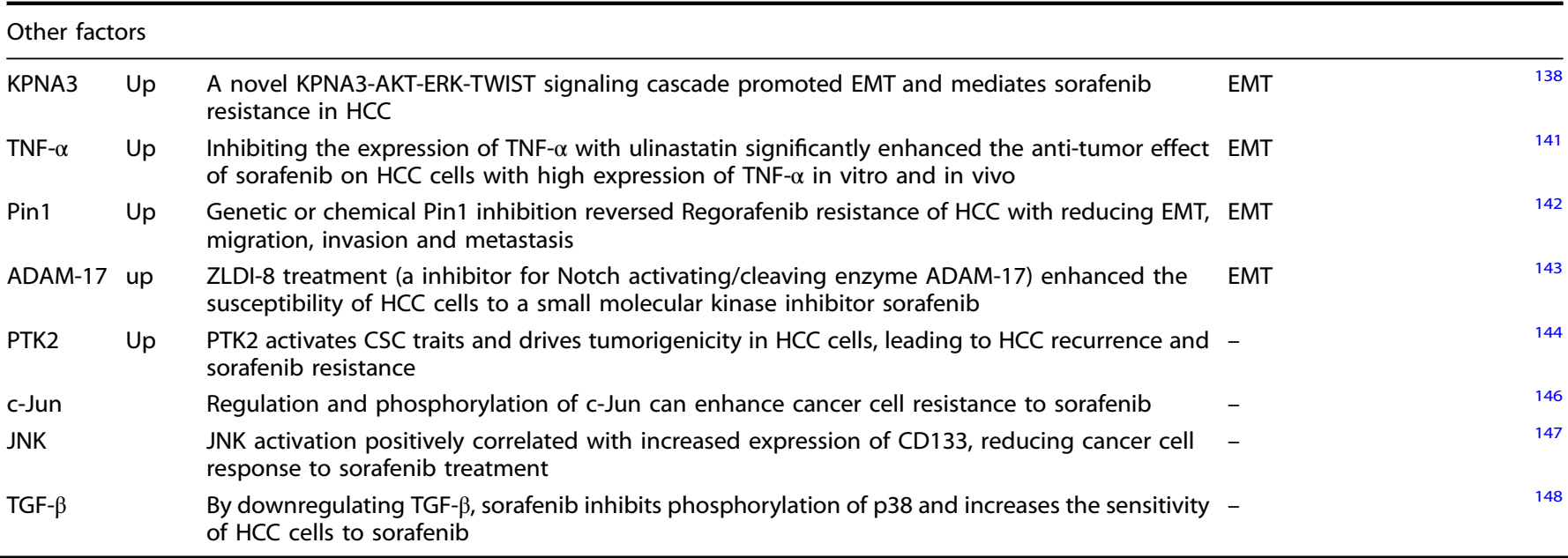

theoretical perspective, the pharmacokinetic characteristics of immunotherapies make them rational choices in HCC that will not cause serious hepatotoxicity. ${ }^{170}$ Therefore, the combination of immunotherapy drugs and sorafenib to treat HCC in the late stage represents a novel therapeutic approach. Wang et al. ${ }^{171}$ reported that a combination of sorafenib and an anti-PD-L1 monoclonal antibody $(\mathrm{mAb})$ can be used to treat HCC. According to Chen and colleagues, ${ }^{114}$ anti-PD-1 therapy enhanced the antitumor immune response of HCC models. Immunotherapy combinations containing anti-PD-1 antibodies plus sorafenib showed good performance only when they contained another drug to simultaneously target the immunosuppressive and hypoxic characteristics of the microenvironment (e.g., an additional CXCR4-inhibiting element). Recent in vivo and in vitro results suggest that targeting CD47 with combinations including sorafenib results in a beneficial effect on patients with HCC. ${ }^{172}$ However, the effective use immunotherapy in HCC must fully consider the HCC-specific immune microenvironment and response.

Conclusions and future perspectives

Resistance to systemic sorafenib therapy emerges early in HCC. To enhance the antitumor effect induced by sorafenib, it is urgent to understand its potential mechanism and identify therapeutic targets. This study comprehensively summarized the molecular, cellular, and microenvironmental mechanisms that are likely to collectively facilitate sorafenib resistance in HCC. Epigenetic biological processes, transport processes, regulated cell death, and the tumor microenvironment have been shown to be related to resistance to sorafenib. To maintain the efficacy of sorafenib against HCC, we need to examine treatments for initial or acquired drug resistance.

\section{ACKNOWLEDGEMENTS}

This work was supported by grants from the National Natural Science Key Foundation of China (grant No. 81530048) and the National Natural Youth Fund (grant No. 81902485).

\section{AUTHOR CONTRIBUTIONS}

There are three first authors of this manuscript, and they have equally contributed to this project. W.W.T., Z.Y.C., and W.L.Z. were responsible for gathering information on the related research and designing the review. Y.C., B.Z., F.W., Q.W., S.J.W., and D.W.R. were responsible for creating the figures. Furthermore, we have three corresponding authors for this manuscript. X.W. contributed to information interpretation, editing and critical revision of the manuscript. F.P.R. and E.N.T. contributed to the study design and critical revision of the manuscript. F.P.R., E.N.T., and X.H.W. were also responsible for handling the revisions and resubmission of revised manuscripts. All authors read and approved the final manuscript.

\section{ADDITIONAL INFORMATION}

Competing interests: The authors declare no competing interests.

\section{REFERENCES}

1. Torre, L. A. et al. Global cancer statistics, 2012. Cancer J. Clin. 65, 87-108 (2015).

2. Llovet, J. M. et al. Hepatocellular carcinoma. Nat. Rev. Dis. Prim. 2, 16018 (2016).

3. Forner, A., Reig, M. \& Bruix, J. Hepatocellular carcinoma. Lancet 391, 1301-1314 (2018).

4. Li, L. \& Wang, H. Heterogeneity of liver cancer and personalized therapy. Cancer Lett. 379, 191-197 (2016).

5. Llovet, J. M. et al. Sorafenib in advanced hepatocellular carcinoma. N. Engl. J. Med. 359, 378-390 (2008).

6. Wilhelm, S. M. et al. BAY 43-9006 exhibits broad spectrum oral antitumor activity and targets the RAF/MEK/ERK pathway and receptor tyrosine kinases involved in tumor progression and angiogenesis. Cancer Res. 64, 7099-109 (2004).

7. Vogel, A. \& Saborowski, A. Current strategies for the treatment of intermediate and advanced hepatocellular carcinoma. Cancer Treat. Rev. 82, 101946 (2020).

8. Cheng, A. L. et al. Efficacy and safety of sorafenib in patients in the Asia-Pacific region with advanced hepatocellular carcinoma: a phase III randomised, doubleblind, placebo controlled trial. Lancet Oncol. 10, 25-34 (2009).

9. Kudo, M. et al. Lenvatinib versus sorafenib in first-line treatment of patients with unresectable hepatocellular carcinoma: a randomised phase 3 non-inferiority trial. Lancet 391, 1163-117 (2018).

10. Bruix, J. et al. Regorafenib for patients with hepatocellular carcinoma who progressed on sorafenib treatment (RESORCE): a randomised, double-blind, placebo-controlled, phase 3 trial. Lancet 389, 56-66 (2017).

11. Zhu, A. X. et al. Ramucirumab versus placebo as second-line treatment in patients with advanced hepatocellular carcinoma following first-line therapy with sorafenib (REACH): a randomised, double-blind, multicentre, phase 3 trial. Lancet Oncol. 16, 859-870 (2015).

12. Abou-Alfa, G. K. et al. Cabozantinib in patients with advanced and progressing hepatocellular carcinoma. N. Engl. J. Med. 379, 54-63 (2018).

13. Liu, X. \& Qin, S. Immune checkpoint inhibitors in hepatocellular carcinoma: opportunities and challenges. Oncologist 24, S3-S10 (2019).

14. Trojan, J. \& Waidmann, O. Role of regorafenib as second-line therapy and landscape of investigational treatment options in advanced hepatocellular carcinoma. J. Hepatocell. Carcinoma 3, 31-36 (2016).

15. Cheng, A. L. IMbrave150: efficacy and safety results from a ph III study evaluating atezolizumab (atezo) + bevacizumab (bev) vs sorafenib (Sor) as first treatment (tx) for patients (pts) with unresectable hepatocellular carcinoma (HCC). Ann. Oncol. 30, ix186-ix187 (2019).

16. Sui, Z. G. et al. Sorafenib plus capecitabine for patients with advanced hepatocellular caicinoma. China Pharm. 19, 848-849 (2008). 
17. Colagrande, S. et al. Advanced hepatocellular carcinoma and sorafenib: diagnosis, indications, clinical and radiological follow-up. World J. Hepatol. 7, 1041-1053 (2015).

18. Ge, R. et al. Epigenetic modulations and lineage plasticity in advanced prostate cancer. Ann. Oncol. 31, 470-479 (2020).

19. $\mathrm{Li}$, S. et al. Epigenetics/Epigenomics of triterpenoids in cancer prevention and in health. Biochem. Pharmacol. 175, 113890 (2020).

20. Baharudin, R. et al. Epigenetics of SFRP1: the dual roles in human cancers. Cancers 12, 445 (2020).

21. Li, W. et al. LncRNA SNHG1 contributes to sorafenib resistance by activating the Akt pathway and is positively regulated by miR-21 in hepatocellular carcinoma cells. J. Exp. Clin. Cancer Res. 38, 183 (2019).

22. Chen, S. \& Xia, X. Long noncoding RNA NEAT1 suppresses sorafenib sensitivity of hepatocellular carcinoma cells via regulating miR-335-c-Met. J. Cell. Physiol. 234, 14999-15009 (2019).

23. Schultheiss, C. S. et al. The long non-coding RNA H19 suppresses carcinogenesis and chemoresistance in hepatocellular carcinoma. Cell Stress 1, 37-54 (2017).

24. Jin, W. et al. Long non-coding RNA TUC338 is functionally involved in sorafenibsensitized hepatocarcinoma cells by targeting RASAL1. Oncol. Rep. 37, 273-280 (2017).

25. Tang, S. et al. An artificial IncRNA targeting multiple miRNAs overcomes sorafenib resistance in hepatocellular carcinoma cells. Oncotarget 7, 73257-73269 (2016).

26. Takahashi, K. et al. Extracellular vesicle-mediated transfer of long non-coding RNA ROR modulates chemosensitivity in human hepatocellular cancer. FEBS Open Bio 4, 458-467 (2014).

27. Quagliata, L. et al. High expression of HOXA13 correlates with poorly differentiated hepatocellular carcinomas and modulates sorafenib response in in vitro models. Lab. Invest. 98, 95-105 (2018).

28. Zhang, T. et al. SNHG3 correlates with malignant status and poor prognosis in hepatocellular carcinoma. Tumour Biol. 37, 2379-2385 (2016).

29. $\mathrm{Ye}$, J. et al. Long noncoding RNA SNHG16 induces sorafenib resistance in hepatocellular carcinoma cells through sponging miR-140-5p. Onco Targets Ther. 12, 415-422 (2019).

30. Sui, C. et al. LncRNA FOXD2-AS1 as a competitive endogenous RNA against miR150-5p reverses resistance to sorafenib in hepatocellular carcinoma. J. Cell Mol. Med. 23, 6024-6033 (2019).

31. Wang, Z. et al. Anti-GPC3 antibody tagged cationic switchable lipid-based nanoparticles for the co-delivery of anti-miRNA27a and sorafenib in liver cancers. Pharm. Res. 36, 145 (2019).

32. Zhang, $M$ et al. MiR-374b re-sensitizes hepatocellular carcinoma cells to sorafenib therapy by antagonizing PKM2-mediated glycolysis pathway. Am. J. Cancer Res. 9, 765-778 (2019).

33. Jiang, X. M. et al. MicroRNA-19a-3p promotes tumor metastasis and chemoresistance through the PTEN/Akt pathway in hepatocellular carcinoma. Biomed. Pharmacother. 105, 1147-1154 (2018).

34. Callegari, E. et al. MiR-199a-3p modulates MTOR and PAK4 pathways and inhibits tumor growth in a hepatocellular carcinoma transgenic mouse model. Mol. Ther. Nucleic Acids 11, 485-493 (2018).

35. Pollutri, D. et al. The epigenetically regulated miR-494 associates with stem-cell phenotype and induces sorafenib resistance in hepatocellular carcinoma. Cell Death Dis. 9, 4 (2018).

36. Lu, A. Q. et al. Upregulation of miR-137 reverses sorafenib resistance and cancerinitiating cell phenotypes by degrading ANT2 in hepatocellular carcinoma. Oncol. Rep. 37, 2071-2078 (2017).

37. Fornari, F. et al. In hepatocellular carcinoma miR-221 modulates sorafenib resistance through inhibition of caspase-3-mediated apoptosis. Clin. Cancer Res. 23, 3953-3965 (2017)

38. Potenza, N. et al. MicroRNA-125a-5p Is a downstream effector of sorafenib in its antiproliferative activity toward human hepatocellular carcinoma cells. J. Cell Physiol. 232, 1907-1913 (2017).

39. Xu, J. et al. The miR-367-3p increases sorafenib chemotherapy efficacy to suppress hepatocellular carcinoma metastasis through altering the androgen receptor signals. EBioMedicine 12, 55-67 (2016)

40. Azumi, J. et al. miR-181a induces sorafenib resistance of hepatocellular carcinoma cells through downregulation of RASSF1 expression. Cancer Sci. 107, 1256-1262 (2016).

41. Lou, G. et al. Exosomes derived from miR-122-modified adipose tissue-derived MSCs increase chemosensitivity of hepatocellular carcinoma. J. Hematol. Oncol. 8, 122 (2015).

42. Wang, $\mathrm{H}$. et al. MicroRNA-591 functions as a tumor suppressor in hepatocellular carcinoma by lowering drug resistance through inhibition of far-upstream element-binding protein 2-mediated phosphoinositide 3-Kinase/Akt/mammalian target of rapamycin axis. Pharmacology 104, 173-186 (2019).
43. Dietrich, P. et al. Wild type Kirsten rat sarcoma is a novel microRNA-622regulated therapeutic target for hepatocellular carcinoma and contributes to sorafenib resistance. Gut 67, 1328-1341 (2018).

44. Kabir, T. D. et al. A microRNA-7/growth arrest specific 6/TYRO3 axis regulates the growth and invasiveness of sorafenib-resistant cells in human hepatocellular carcinoma. Hepatology 67, 216-231 (2018)

45. Mendez-Blanco, C. et al. Stabilization of hypoxia-inducible factors and BNIP3 promoter methylation contribute to acquired sorafenib resistance in human hepatocarcinoma cells. Cancers 11, 1984 (2019).

46. Wong, T. L. et al. CRAF methylation by PRMT6 regulates aerobic glycolysis-driven hepatocarcinogenesis via ERK-dependent PKM2 nuclear relocalization and activation. Hepatology 71, 1279-1296 (2019).

47. Fan, Z. et al. PTK2 promotes cancer stem cell traits in hepatocellular carcinoma by activating Wnt/ $\beta$-catenin signaling. Cancer Lett. 450, 132-143 (2019).

48. Gailhouste, L. et al. Differentiation therapy by epigenetic reconditioning exerts antitumor effects on liver cancer cells. Mol. Ther. 26, 1840-1854 (2018).

49. Wang, T. et al. Epigenetic restriction of Hippo signaling by MORC2 underlies stemness of hepatocellular carcinoma cells. Cell Death Differ. 25, 2086-2100 (2018).

50. Liu, Y. et al. Demethylation-induced overexpression of Shc3 drives c-Rafindependent activation of MEK/ERK in HCC. Cancer Res. 78, 2219-2232 (2018).

51. Liu, J. et al. Targeting the PD-L1/DNMT1 axis in acquired resistance to sorafenib in human hepatocellular carcinoma. Oncol. Rep. 38, 899-907 (2017).

52. Abeni, E. et al. Sorafenib induces variations of the DNA methylome in HA22T/ VGH human hepatocellular carcinoma-derived cells. Int. J. Oncol. 51, 128-144 (2017).

53. Huo, Q. et al. Dysfunction of IKZF1/MYC/MDIG axis contributes to liver cancer progression through regulating H3K9me3/p21 activity. Cell Death Dis. 8, e2766 (2017).

54. He, R. Z., Luo, D. X. \& Mo, Y. Y. Emerging roles of IncRNAs in the posttranscriptional regulation in cancer. Genes Dis. 6, 6-15 (2019).

55. Lee, D. D. et al. DNA methylation of the TERT promoter and its impact on human cancer. Curr. Opin. Genet. Dev. 60, 17-24 (2020).

56. Hudlikar, R. R. et al. Triterpenoid corosolic acid modulates global CpG methylation and transcriptome of tumor promotor TPA induced mouse epidermal JB6 P+ cells. Chem. Biol. Interact. 321, 109025 (2020).

57. Mancini, M. et al. HP1s modulate the S-adenosyl methionine pathway in liver cancer cells. Biochem J. 477, 1033-1047 (2020).

58. Beretta, G. L. et al. Overcoming $A B C$ transporter-mediated multidrug resistance: the dual role of tyrosine kinase inhibitors as multitargeting agents. Eur. J. Med. Chem. 142, 271-289 (2017).

59. Durmus, S., Hendrikx, J. J. \& Schinkel, A. H. Apical ABC transporters and cancer chemotherapeutic drug disposition. Adv. Cancer Res. 125, 1-41 (2015).

60. Arrighetti, N. et al. Exosome-like nanovectors for drug delivery in cancer. Curr. Med. Chem. 26, 6132-6148 (2019).

61. Gowda, R. et al. The role of exosomes in metastasis and progression of melanoma. Cancer Treat. Rev. 85, 101975 (2020).

62. Di Giacomo, S. et al. Chemosensitization of hepatocellular carcinoma cells to sorafenib by $\beta$-caryophyllene oxide-induced inhibition of $A B C$ export pumps. Arch. Toxicol. 93, 623-634 (2019).

63. Wang, $\mathrm{H}$. et al. CSN5 silencing reverses sorafenib resistance of human hepatocellular carcinoma HepG2 cells. Mol. Med Rep. 12, 3902-3908 (2015)

64. Chow, A. K. et al. The enhanced metastatic potential of hepatocellular carcinoma (HCC) cells with sorafenib resistance. PLOS ONE 8, e78675 (2013).

65. Wang, G. et al. Exosomal MiR-744 inhibits proliferation and sorafenib chemoresistance in hepatocellular carcinoma by targeting PAX2. Med. Sci. Monit. 25, 7209-7217 (2019).

66. $\mathrm{Qu}, \mathrm{Z}$. et al. Exosomes derived from HCC cells induce sorafenib resistance in hepatocellular carcinoma both in vivo and in vitro. J. Exp. Clin. Cancer Res. 35, 159 (2016).

67. Takahashi, K. et al. Involvement of extracellular vesicle long noncoding RNA (linc-VLDLR) in tumor cell responses to chemotherapy. Mol. Cancer Res. 12, 1377-1387 (2014).

68. $\mathrm{Li}, \mathrm{H}$ et al. Exosomes derived from siRNA against GRP78 modified bone-marrowderived mesenchymal stem cells suppress Sorafenib resistance in hepatocellular carcinoma. J. Nanobiotechnol. 16, 103 (2018).

69. Shi, S. et al. Dendritic cells pulsed with exosomes in combination with PD-1 antibody increase the efficacy of sorafenib in hepatocellular carcinoma model. Transl. Oncol. 11, 250-258 (2018).

70. Jayaseelan, V. P. Emerging role of exosomes as promising diagnostic tool for cancer. Cancer Gene Ther. (2019).

71. Tian, W., Liu, S. \& Li, B. Potential role of exosomes in cancer metastasis. Biomed. Res. Int. 2019, 4649705 (2019).

72. Safaei, R. et al. Abnormal lysosomal trafficking and enhanced exosomal export of cisplatin in drug-resistant human ovarian carcinoma cells. Mol. Cancer Ther. 4 , 1595-604 (2005). 
73. Lee, Y. \& Overholtzer, M. After-death functions of cell death. Yale J. Biol. Med. 92, 687-694 (2019).

74. Galimov, E. R., Lohr, J. N. \& Gems, D. When and how can death be an adaptation? Biochemistry 84, 1433-1437 (2019).

75. Shi, Y. H. et al. Targeting autophagy enhances sorafenib lethality for hepatocellular carcinoma via ER stress-related apoptosis. Autophagy 7, 1159-1172 (2011).

76. Tai, W. T. et al. Mcl-1-dependent activation of Beclin 1 mediates autophagic cell death induced by sorafenib and SC-59 in hepatocellular carcinoma cells. Cell Death Dis. 4, e485 (2013).

77. Zhai, B. et al. Inhibition of Akt reverses the acquired resistance to sorafenib by switching protective autophagy to autophagic cell death in hepatocellular carcinoma. Mol. Cancer Ther. 13, 1589-1598 (2014).

78. Yuan, H. et al. Inhibition of autophagy significantly enhances combination therapy with sorafenib and HDAC inhibitors for human hepatoma cells. World J. Gastroenterol. 20, 4953-4962 (2014).

79. Ling, S. et al. Combination of metformin and sorafenib suppresses proliferation and induces autophagy of hepatocellular carcinoma via targeting the mTOR pathway. Int. J. Oncol. 50, 297-309 (2017).

80. Luo, T. et al. PSMD10/gankyrin induces autophagy to promote tumor progression through cytoplasmic interaction with ATG7 and nuclear transactivation of ATG7 expression. Autophagy 12, 1355-1371 (2016).

81. Fischer, T. D. et al. Role of autophagy in differential sensitivity of hepatocarcinoma cells to sorafenib. World J. Hepatol. 6, 752-758 (2014).

82. $\mathrm{Wu}, \mathrm{F}$. Q. et al. ADRB2 signaling promotes $\mathrm{HCC}$ progression and sorafenib resistance by inhibiting autophagic degradation of HIF1a. J. Hepatol. 65, 314-324 (2016).

83. Yu., S. et al. B-cell lymphoma-2-associated transcription factor 1 is overexpressed and contributes to sorafenib resistance in hepatocellular carcinoma. Hepatol. Res. 49, 1329-1340 (2019).

84. Dai, N. et al. Capsaicin and sorafenib combination treatment exerts synergistic anti-hepatocellular carcinoma activity by suppressing EGFR and PI3K/Akt/mTOR signaling. Oncol. Rep. 40, 3235-3248 (2018).

85. Lu, S. et al. CD24 regulates sorafenib resistance via activating autophagy in hepatocellular carcinoma. Cell Death Dis. 9, 646 (2018).

86. $\mathrm{He}, \mathrm{C}$. et al. MiR-21 mediates sorafenib resistance of hepatocellular carcinoma cells by inhibiting autophagy via the PTEN/Akt pathway. Oncotarget $\mathbf{6}$, 28867-28881 (2015).

87. Stiuso, P. et al. MicroRNA-423-5p promotes autophagy in cancer cells and is increased in serum from hepatocarcinoma patients treated with sorafenib. Mol. Ther. Nucleic Acids 4, e233 (2015).

88. Dai, E. et al. AIFM2 blocks ferroptosis independent of ubiquinol metabolism. Biochem. Biophys. Res. Commun. 523, 966-971 (2020).

89. Zhang, Z. et al. RNA-binding protein ZFP36/TTP protects against ferroptosis by regulating autophagy signaling pathway in hepatic stellate cells. Autophagy 1-24 (2019).

90. Sun, X. et al. Metallothionein-1G facilitates sorafenib resistance through inhibition of ferroptosis. Hepatology 64, 488-500 (2016).

91. Zhang, Z. et al. Activation of ferritinophagy is required for the RNA-binding protein ELAVL1/HuR to regulate ferroptosis in hepatic stellate cells. Autophagy 14, 2083-2103 (2018).

92. Janku, F. et al. Autophagy as a target for anticancer therapy. Nat. Rev. Clin. Oncol. 8, 528-539 (2011).

93. Mizushima, N. Autophagy: process and function. Genes Dev. 21, 2861-73 (2007).

94. Shintani, T. \& Klionsky, D. J. Autophagy in health and disease: a double-edged sword. Science 306, 990-995 (2004).

95. White, E. \& DiPaola, R. S. The double-edged sword of autophagy modulation in cancer. Clin. Cancer Res. 15, 5308-5316 (2009).

96. Liu, J. et al. Autophagy, a double-edged sword in anti-angiogenesis therapy. Med. Oncol. 33, 10 (2016).

97. Thorburn, A. Autophagy and its effects: making sense of double-edged swords. PLOS Biol. 12, e1001967 (2014).

98. Nie, J. et al. Role of ferroptosis in hepatocellular carcinoma. J. Cancer Res. Clin. Oncol. 144, 2329-2337 (2018)

99. Louandre, C. et al. Iron-dependent cell death of hepatocellular carcinoma cells exposed to sorafenib. Int. J. Cancer 133, 1732-1742 (2013).

100. Lachaier, E. et al. Sorafenib induces ferroptosis in human cancer cell lines originating from different solid tumors. Anticancer Res. 34, 6417-6422 (2014).

101. Liang, Y. et al. Hypoxia-mediated sorafenib resistance can be overcome by EF24 through Von Hippel-Lindau tumor suppressor-dependent HIF-1a inhibition in hepatocellular carcinoma. Hepatology 57, 1847-1857 (2013).

102. Prieto-Domínguez, N. et al. Melatonin enhances sorafenib actions in human hepatocarcinoma cells by inhibiting mTORC1/p70S6K/HIF-1a and hypoxiamediated mitophagy. Oncotarget 8, 91402-91414 (2017).
103. Xu, H. et al. MiR-338-3p inhibits hepatocarcinoma cells and sensitizes these cells to sorafenib by targeting hypoxia-induced factor 1a. PLOS ONE 9, e115565 (2014).

104. Li, S. et al. Genistein suppresses aerobic glycolysis and induces hepatocellular carcinoma cell death. Br. J. Cancer 117, 1518-1528 (2017).

105. Liu, F. et al. Targeting hypoxia-inducible factor-2a enhances sorafenib antitumor activity via $\beta$-catenin/C-Myc-dependent pathways in hepatocellular carcinoma. Oncol. Lett. 10, 778-784 (2015).

106. Ma, L. et al. 2-Methoxyestradiol synergizes with sorafenib to suppress hepatocellular carcinoma by simultaneously dysregulating hypoxia-inducible factor- 1 and -2. Cancer Lett. 355, 96-105 (2014).

107. Zhao, D. et al. Upregulation of HIF-2a induced by sorafenib contributes to the resistance by activating the TGF-a/EGFR pathway in hepatocellular carcinoma cells. Cell Signal 26, 1030-1039 (2014)

108. $\mathrm{Xu}$, J. et al. Increasing AR by HIF-2a inhibitor (PT-2385) overcomes the sideeffects of sorafenib by suppressing hepatocellular carcinoma invasion via alteration of pSTAT3, pAKT and pERK signals. Cell Death Dis. 8, e3095 (2017).

109. Casadei Gardini, A. et al. Metformin and insulin impact on clinical outcome in patients with advanced hepatocellular carcinoma receiving sorafenib: validation study and biological rationale. Eur. J. Cancer 86, 106-114 (2017).

110. Song, Z. et al. HIF-1a-induced RIT1 promotes liver cancer growth and metastasis and its deficiency increases sensitivity to sorafenib. Cancer Lett. 460, 96-107 (2019).

111. Long, Q. et al. PFKFB3/HIF-1a feedback loop modulates sorafenib resistance in hepatocellular carcinoma cells. Biochem. Biophys. Res. Commun. 513, 642-650 (2019).

112. Fu, Q. H. et al. LB-100 sensitizes hepatocellular carcinoma cells to the effects of sorafenib during hypoxia by activation of Smad3 phosphorylation. Tumour Biol. 37, 7277-7286 (2016).

113. Yao, W. et al. A natural CCR2 antagonist relieves tumor-associated macrophagemediated immunosuppression to produce a therapeutic effect for liver cancer. EBioMedicine 22, 58-67 (2017).

114. Chen, Y. et al. CXCR4 inhibition in tumor microenvironment facilitates antiprogrammed death receptor-1 immunotherapy in sorafenib-treated hepatocellular carcinoma in mice. Hepatology 61, 1591-602 (2015).

115. Dong, N. et al. M2 macrophages mediate sorafenib resistance by secreting HGF in a feed-forward manner in hepatocellular carcinoma. Br. J. Cancer 121, 22-33 (2019).

116. Zhou, S. L. et al. Tumor-associated neutrophils recruit macrophages and Tregulatory cells to promote progression of hepatocellular carcinoma and resistance to sorafenib. Gastroenterology 150, 1646-1658 (2016).

117. Chen, J. et al. Sorafenib-resistant hepatocellular carcinoma stratified by phosphorylated ERK activates PD-1 immune checkpoint. Oncotarget 7, 41274-41284 (2016).

118. Li, C. et al. Sorafenib attenuated the function of natural killer cells infiltrated in HCC through inhibiting ERK1/2. Int. Immunopharmacol. 76, 105855 (2019).

119. Tao, Z. et al. Targeting the YB-1/PD-L1 axis to enhance chemotherapy and antitumor immunity. Cancer Immunol. Res. 7, 1135-1147 (2019).

120. Lin, X. et al. Silencing MYH9 blocks HBx-induced GSK3beta ubiquitination and degradation to inhibit tumor stemness in hepatocellular carcinoma. Signal Transduct. Target Ther. 5, 13 (2020).

121. Witt-Kehati, D. Inhibition of pMAPK14 overcomes resistance to sorafenib in hepatoma cells with hepatitis B. Virus 11, 511-517 (2018).

122. Ching, R. H. H. et al. C-terminal truncated hepatitis $B$ virus $X$ protein regulates tumorigenicity, self-renewal and drug resistance via STAT3/Nanog signaling pathway. Oncotarget 8, 23507-23516 (2017).

123. Liu, L. P. et al. Sorafenib inhibits hypoxia-inducible factor-1a synthesis: implications for antiangiogenic activity in hepatocellular carcinoma. Clin. Cancer Res. 18, 5662-5671 (2012).

124. $\mathrm{Xu}, \mathrm{M}$. et al. Sorafenib suppresses the rapid progress of hepatocellular carcinoma after insufficient radiofrequency ablation therapy: an experiment in vivo. Acta Radio. 54, 199-204 (2013).

125. Xu, M. et al. Sorafenib blocks the HIF-1a/VEGFA pathway, inhibits tumor invasion, and induces apoptosis in hepatoma cells. DNA Cell Biol. 33, 275-281 (2014).

126. Yeh, C. C. et al. Integrated stable isotope labeling by amino acids in cell culture (SILAC) and isobaric tags for relative and absolute quantitation (iTRAQ) quantitative proteomic analysis identifies galectin-1 as a potential biomarker for predicting sorafenib resistance in liver cancer. Mol. Cell Proteom. 14, 1527-1545 (2015).

127. Méndez-Blanco, C. et al. Sorafenib resistance in hepatocarcinoma: role of hypoxia-inducible factors. Exp. Mol. Med 50, 134 (2018).

128. Nakamura, K. \& Smyth, M. J. Myeloid immunosuppression and immune checkpoints in the tumor microenvironment. Cell. Mol. Immunol. 17, 1-2 (2019). 
129. Zhu, H. et al. Efficacy of anti-PD-1 antibody SHR-1210 as second-line treatment in hepatocellular carcinoma patient with sorafenib resistance: a case report. Medicine 98, e15755 (2019).

130. Berasain, C. et al. Inflammation and liver cancer: new molecular links. Ann. N. Y. Acad. Sci. 1155, 206-221 (2009).

131. Pattullo, V. Hepatitis B reactivation in the setting of chemotherapy and immunosuppression-prevention is better than cure. World J. Hepatol. 7, 954-967 (2015).

132. D'Angelo, S. et al. Sorafenib and entecavir: the dioscuri of treatment for advanced hepatocellular carcinoma? World J. Gastroenterol. 19, 2141-2143 (2013).

133. Zuo, C. et al. Role of antiviral therapy in reducing recurrence and improving survival in hepatitis B virus-associated hepatocellular carcinoma following curative resection. Oncol. Lett. 9, 527-534 (2015).

134. Descamps, V. et al. The kinase-inhibitor sorafenib inhibits multiple steps of the hepatitis C virus infectious cycle in vitro. Antivir. Res. 118, 93-102 (2015).

135. Cabrera, R. et al. The anti-viral effect of sorafenib in hepatitis C-related hepatocellular carcinoma. Aliment Pharm. Ther. 37, 91-97 (2013).

136. Himmelsbach, K. \& Hildt, E. The kinase inhibitor sorafenib impairs the antiviral effect of interferon alpha on hepatitis C virus replication. Eur. J. Cell Biol. 92, 12-20 (2013).

137. Zhang, Q. B. et al. Suppression of natural killer cells by sorafenib contributes to prometastatic effects in hepatocellular carcinoma. PLOS ONE 8, e55945 (2013).

138. $\mathrm{Hu}, \mathrm{B}$. et al. KPNA3 confers sorafenib resistance to advanced hepatocellular carcinoma via TWIST regulated epithelial-mesenchymal transition. J. Cancer 10, 3914-3925 (2019).

139. Xu, Y. et al. KIAA1199 promotes sorafenib tolerance and the metastasis of hepatocellular carcinoma by activating the EGF/EGFR-dependent epithelialmesenchymal transition program. Cancer Lett. 454, 78-89 (2019).

140. Wu, C. X. et al. Blocking CDK1/PDK1/ $\beta$-Catenin signaling by CDK1 inhibitor RO3306 increased the efficacy of sorafenib treatment by targeting cancer stem cells in a preclinical model of hepatocellular carcinoma. Theranostics $\mathbf{8}$ 3737-3750 (2018)

141. Tan, W. et al. TNF-a is a potential therapeutic target to overcome sorafenib resistance in hepatocellular carcinoma. EBioMedicine 40, 446-456 (2019).

142. Wang, J. et al. Pin1 inhibition reverses the acquired resistance of human hepatocellular carcinoma cells to Regorafenib via the Gli1/Snail/E-cadherin pathway. Cancer Lett. 444, 82-93 (2019).

143. Zhang, Y. et al. Novel ADAM-17 inhibitor ZLDI-8 enhances the in vitro and in vivo chemotherapeutic effects of Sorafenib on hepatocellular carcinoma cells. Cell Death Dis. 9, 743 (2018).

144. Fan, Z. et al. PTK2 promotes cancer stem cell traits in hepatocellular carcinoma by activating Wnt/ $\beta$-catenin signaling. Cancer Lett. 450, 132-143 (2019).

145. Xiao, Y. et al. Androgen receptor (AR)/miR-520f-3p/SOX9 signaling is involved in altering hepatocellular carcinoma (HCC) cell sensitivity to the sorafenib therapy under hypoxia via increasing cancer stem cells phenotype. Cancer Lett. 444, 175-187 (2019).

146. Haga, Y. et al. Overexpression of c-Jun contributes to sorafenib resistance in human hepatoma cell lines. PLoS ONE 12, e0174153 (2017).

147. Hagiwara, S. et al. Activation of JNK and high expression level of CD133 predict a poor response to sorafenib in hepatocellular carcinoma. Br. J. Cancer 106, 1997-2003 (2012).

148. Kang, D. et al. Down-regulation of TGF-beta expression sensitizes the resistance of hepatocellular carcinoma cells to sorafenib. Yonsei Med. J. 58, 899-909 (2017).

149. Wang, Z. et al. Sorafenib-irinotecan sequential therapy augmented the antitumor efficacy of monotherapy in hepatocellular carcinoma cells HepG2. Neoplasma 62, 172-179 (2015).

150. Abou-Alfa, G. K. et al. Doxorubicin plus sorafenib vs doxorubicin alone in patients with advanced hepatocellular carcinoma: a randomized trial. JAMA 304, 2154-2160 (2010)

151. Yang, T. et al. Ultrasound-triggered nanodroplets for targeted co-delivery of sorafenib/doxorubicin for hepatocellular carcinoma therapy. J. Biomed. Nanotechnol. 15, 1881-1896 (2019).

152. Liu, Y. et al. First-line gemcitabine and oxaliplatin (GEMOX) plus sorafenib, followed by sorafenib as maintenance therapy, for patients with advanced hepatocellular carcinoma: a preliminary study. Int. J. Clin. Oncol. 20, 952-9 (2015).

153. Ma, X. et al. NOD2 inhibits tumorigenesis and increases chemosensitivity of hepatocellular carcinoma by targeting AMPK pathway. Cell Death Dis. 11, 174 (2020).
154. $\mathrm{Ng}$, L. et al. Suppression of Slit3 induces tumor proliferation and chemoresistance in hepatocellular carcinoma through activation of GSK3beta/beta-catenin pathway. BMC Cancer 18, 621 (2018).

155. Xue, F. et al. elF5A2 is an alternative pathway for cell proliferation in cetuximabtreated epithelial hepatocellular carcinoma. Am. J. Transl. Res. 8, 4670-4681 (2016).

156. Chen, W. et al. Rapamycin enhances cetuximab cytotoxicity by inhibiting mTORmediated drug resistance in mesenchymal hepatoma cells. Cancer Biol. Ther. 15 992-999 (2014).

157. Geng, J. et al. Combination of cetuximab and rapamycin enhances the therapeutic efficacy in hepatocellular carcinoma. Technol. Cancer Res. Treat. 13, 377-385 (2014).

158. Zhu, A. X. et al. Phase 2 study of cetuximab in patients with advanced hepatocellular carcinoma. Cancer 110, 581-589 (2007).

159. Sanoff, H. K. et al. Phasell study of capecitabine, oxaliplatin, and cetuximab for advanced hepatocellular carcinoma. Gastrointest. Cancer Res. 4, 78-83 (2011).

160. Asnacios, A. et al. Gemcitabine plus oxaliplatin (GEMOX) combined with cetuximab in patients with progressive advanced stage hepatocellular carcinoma: results of a multicenter phase 2 study. Cancer 112, 2733-2739 (2008).

161. Zhu, A. X. et al. SEARCH: a phase III, randomized, double-blind, placebocontrolled trial of sorafenib plus erlotinib in patients with advanced hepatocellular carcinoma. J. Clin. Oncol. 33, 559-566 (2015).

162. Zhu, Y. J. et al. New knowledge of the mechanisms of sorafenib resistance in liver cancer. Acta Pharm. Sin. 38, 614-622 (2017).

163. Zhang, $\mathrm{H}$. et al. Inhibition of the PI3K/Akt signaling pathway reverses sorafenibderived chemo-resistance in hepatocellular carcinoma. Oncol. Lett. 15, 9377-9384 (2018).

164. Ye, L. et al. The PI3K inhibitor copanlisib synergizes with sorafenib to induce cell death in hepatocellular carcinoma. Cell Death Discov. 5, 86 (2019).

165. Bollard, J. et al. Palbociclib (PD-0332991), a selective CDK4/6 inhibitor, restricts tumour growth in preclinical models of hepatocellular carcinoma. Gut 66, 1286-1296 (2017)

166. Reiter, F. P. et al. Predictors of ribociclib-mediated antitumour effects in native and sorafenib-resistant human hepatocellular carcinoma cells. Cell. Oncol. 42, 705-715 (2019).

167. Marozin, S. et al. STAT3 inhibition reduces toxicity of oncolytic VSV and provides a potentially synergistic combination therapy for hepatocellular carcinoma. Cancer Gene Ther. 22, 317-325 (2015).

168. Gedaly, R. et al. PKI-587 and sorafenib targeting PI3K/AKT/mTOR and Ras/Raf/ MAPK pathways synergistically inhibit HCC cell proliferation. J. Surg. Res. 176, 542-548 (2012).

169. Koeberle, D. et al. Sorafenib with or without everolimus in patients with advanced hepatocellular carcinoma (HCC): a randomized multicenter, multinational phase II trial (SAKK 77/08 and SASL 29). Ann. Oncol. 27, 856-861 (2016).

170. Hato, T. et al. Immune checkpoint blockade in hepatocellular carcinoma: current progress and future directions. Hepatology 60, 1776-1782 (2014).

171. Wang, Y. et al. Combinatorial immunotherapy of sorafenib and blockade of programmed death-ligand 1 induces effective natural killer cell responses against hepatocellular carcinoma. Tumour Biol. 36, 1561-1566 (2015).

172. Lo, J. et al. Nuclear factor kappa B-mediated CD47 up-regulation promotes sorafenib resistance and its blockade synergizes the effect of sorafenib in hepatocellular carcinoma in mice. Hepatology 62, 534-545 (2015).

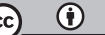

Open Access This article is licensed under a Creative Commons Attribution 4.0 International License, which permits use, sharing, adaptation, distribution and reproduction in any medium or format, as long as you give appropriate credit to the original author(s) and the source, provide a link to the Creative Commons license, and indicate if changes were made. The images or other third party material in this article are included in the article's Creative Commons license, unless indicated otherwise in a credit line to the material. If material is not included in the article's Creative Commons license and your intended use is not permitted by statutory regulation or exceeds the permitted use, you will need to obtain permission directly from the copyright holder. To view a copy of this license, visit http://creativecommons. org/licenses/by/4.0/.

(c) The Author(s) 2020 